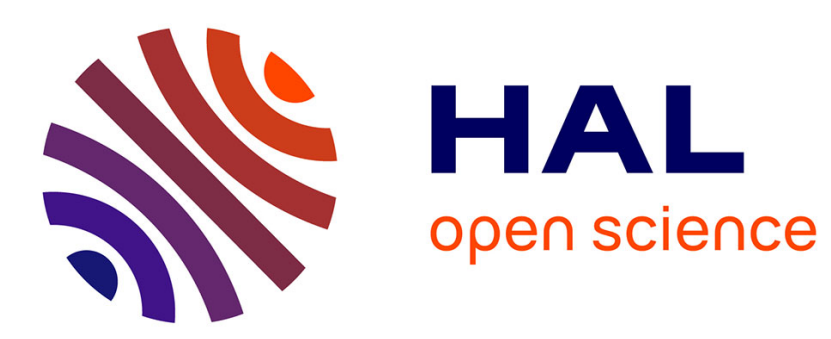

\title{
DOSKIL: A New Mechanism for Controlling Stick-Slip Oscillations in Oil Well Drillstrings
}

Carlos Canudas de Wit, Francisco Rubio, Miguel Corchero

\section{To cite this version:}

Carlos Canudas de Wit, Francisco Rubio, Miguel Corchero. DOSKIL: A New Mechanism for Controlling Stick-Slip Oscillations in Oil Well Drillstrings. IEEE Transactions on Control Systems Technology, 2008, 16 (6), pp.1177-1191. 10.1109/TCST.2008.917873 . hal-00394990

\section{HAL Id: hal-00394990 \\ https://hal.science/hal-00394990}

Submitted on 13 Jun 2009

HAL is a multi-disciplinary open access archive for the deposit and dissemination of scientific research documents, whether they are published or not. The documents may come from teaching and research institutions in France or abroad, or from public or private research centers.
L'archive ouverte pluridisciplinaire HAL, est destinée au dépôt et à la diffusion de documents scientifiques de niveau recherche, publiés ou non, émanant des établissements d'enseignement et de recherche français ou étrangers, des laboratoires publics ou privés. 


\title{
DOSKIL: A New Mechanism for Controlling Stick-Slip Oscillations in Oil Well Drillstrings
}

\author{
Carlos Canudas-de-Wit \\ Laboratoire d'Automatique de Grenoble, INPG-CNRS, Grenoble, FRANCE. \\ Email: carlos.canudas-de-wit@inpg.fr \\ Francisco R. Rubio \\ Department of Automatic Control, University of Seville, Seville, SPAIN \\ Email: rubio@us.es \\ Miguel Angel Corchero \\ Department of Automatic Control, University of Seville, Seville, SPAIN \\ Email: macperu@us.es
}

\begin{abstract}
Limit cycles occurring in oil well drillstrings result from the interaction between the drill bit and the rock during drilling operations. In this paper we propose to use the weight on the bit (WoB) force as an additional control variable to extinguish limit cycles when they occur. An approximate analysis based on the bias describing function and completed with some simulations, provides good evidence that the rotational dynamics of the oil well drillstring displays such a behavior. In particular, we propose an adaptation law for the WoB named D-OKILL mechanisms, which results from a variant of the oscillation killer (OSKIL) mechanism studied in detail in [6].

In opposition to the heuristic control structure proposed in [7], we show that the new Weight on Bit $\left(W_{o B}\right)$ control law results in a globally asymptotically stable closed loop-system. Simulations applying the D-OSKIL mechanism show that the stick-slip oscillations can be eliminated without requiring a re-design of the velocity rotary-table control.
\end{abstract}

\section{INTRODUCTION}

Oil well drillstrings (see Figure 1) are systems which present interesting features from the dynamical and control viewpoints as they pose many challenging technological problems [26], [34]. The application of dynamic analysis and control techniques in a drilling system can lead to conclusions that allow us to propose new recommendations for drilling operations, drillstring design and control algorithm, which would produce economic benefits through a mix of lower development costs, higher production rates and improved recovery. Particularly, the presence of stickslip self-excited oscillations at the bottom part of the drillstrings as well as decreasing service life of drillstrings and downhole equipment, has drawn the attention of the control community in the last decade. The elimination 


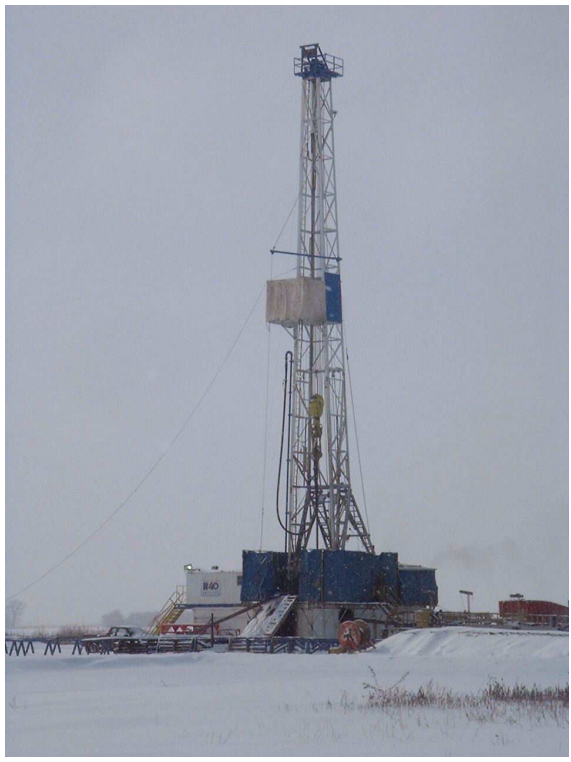

a)

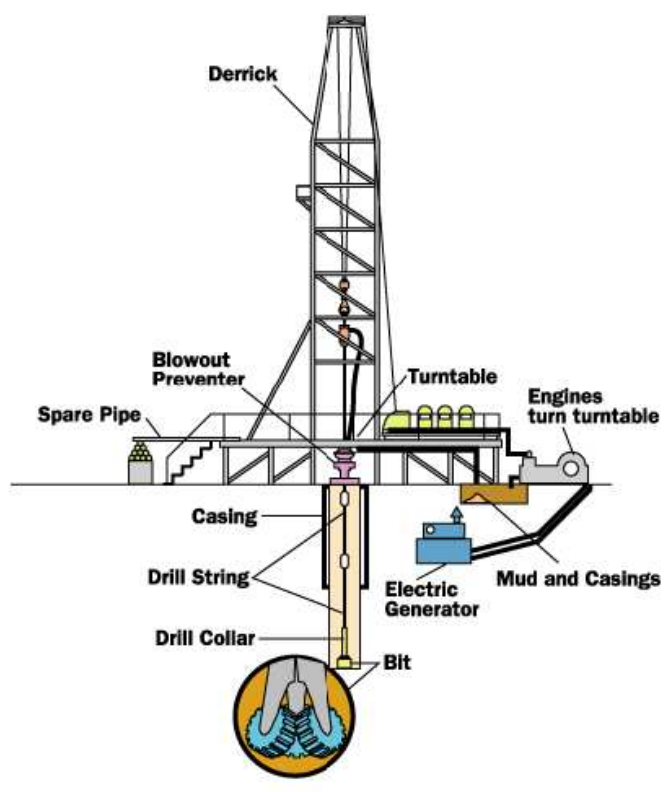

b)

Fig. 1. Oil drilling system in the field (a). Basic scheme of a vertical drilling system.(b)

of this kind of oscillations is a challenge for drillers and scientists since it can provide important cost savings in drilling operations, in terms on money and exploitation time [21].

Different oscillations affecting the drillstring behavior are unavoidable. The occurrence of self-excited stick-slip vibrations (i.e., the top of the drillstring rotates with a constant rotary speed, whereas the bit (cutting device) rotary speed varies between zero and up to six times the rotary speed measured at the surface) as a common and damaging phenomena in drillstring systems has been highly described and analyzed in recent years, drawing the attention of the control community. For more information about drillstring oscillations and stick-slip phenomenon in oil well drillstrings, please see [14], [21], [24], [31].

Some causes of stick-slip oscillations are backlash between contacting parts, hysteresis, nonlinear damping and geometrical imperfections which are very difficult to model. However, the main cause of such vibrations in drillstrings is the friction appearing by contact with the rock formation [3], [17]. Consequently, a model describing the drillstring behavior should include a bit-rock friction torque model adequate enough to properly reproduce this effect.

Many ways of reducing these vibrations have been proposed, both from practical and theoretical viewpoints.

Historically, the experience of drillers has revealed that the manipulation of different drilling parameters (increasing the rotary speed, decreasing the weight-on-bit $(W o B)$, modifying the drilling mud characteristics, introducing an additional friction at the bit [25], etc) is an effective strategy to suppress stick-slip motion [28]. However, this strategy depends too much on the personal skills of each drilling technician to be really effective. 
Usually, drilling systems are velocity-controlled to make them rotate at a constant velocity, but no specifications about vibration suppression or damping are considered. Another control option would be introducing new regulation methodologies (active, passive) in the loop, specifically aimed to compensate for drillstring vibrations. Among those, the following examples can be pointed out:

- The so-called Soft Torque Rotary System (STRS) [13] [28], that is a torque feedback at the top of the drillstring which makes the system behave in a "softer" way rather than as a fixed heavy flywheel, so that the torsional waves arriving at the surface are absorbed, breaking the harmful cycling motion.

- Introducing a vibration absorber at the top of the drillstring [15] which follows the same approach given in [13] and [28].

- Introducing a PID controller structure at the surface in order to control the rotary speed [1], [23], [24], [25].

- Using robust controllers, like the linear $H_{\infty}$ control proposed in [29], to suppress stick-slip motion at the bit.

- Using a controller based on an input-state feedback linearization of the nonlinear friction torque [2].

However, few works have provided a formal stability analysis of their proposed control strategies. For instance, analysis of the dynamical behavior of drillstring under vibrations has been explored in [1] and linear approximations to stability of controlled drillstring has been studied in [24].

The value of the system weight measured at the bottom part, called Weight on Bit $\left(W_{o B}\right)$, has been proved to be an important parameter in the occurrence and possible avoidance of stick-slip oscillations (see [23] and [28]).

Efficient drilling operation requires a certain amount of force $\left(W_{o B}\right)$ that may be incompatible with the low force range which may avoid stick-slip oscillations. This tradeoff between force magnitudes, provides a first indication that a regulation strategy of $W_{o B}$ seems to be necessary to maintain a good drilling operation, thus, avoiding such oscillations (see [7] and [24]).

This paper is focused on the problem of stick-slip oscillations produced at the bottom-hole assembly (BHA). The main idea is to use the weight on the bit $(W o B)$ force as an additional control variable. In particular we adapt the oscillation killer (OSKIL) mechanism studied in [6], to the oil well drillstring systems (named here D-OSKIL ${ }^{1}$ ) which has been shown to be particularly adapted for nonlinear systems displaying a local stable region with a stable limit set outside this local domain.

An approximate analysis based on the bias describing function provides good evidence that the rotational dynamics of the oil well drillstring display a similar behavior pattern. This analysis, although approximate, also gives a good intuition in the way that the $W o B$ needs to be modified to suppress oscillations. An important property of the proposed D-OSKIL mechanism is that it allows recovering the nominal operation condition (the $W o B$ recovers its nominal drilling value) while oscillations are suppressed.

In opposition to the heuristic control structure proposed in [7], we show that the new proposed Weight on Bit $\left(W_{o B}\right)$ control law results in a globally asymptotically stable closed loop-system. Therefore, the D-OSKIL mechanism eliminates the stick-slip oscillations without requiring a re-design of the velocity rotary-table control.

${ }^{1} \mathrm{D}$-OSKIL stands for Drilling oscillation killer mechanism. 
The paper is organized as follows. In Section II the basics about drillstring dynamics and vibrations are briefly introduced. In Section III, the drillstring model used in the paper is presented. The control loop used to regulate rotational velocity of the set and the a priori behavior of the closed loop system are shown in Sections IV and V, respectively. In Section VI, a Describing Function based analysis is made in order to obtain some information about stick-slip oscillations in the system, and next, in Section VII, the control mechanism named D-OSKIL obtained from the conclusions of the previous analysis is presented. In Section VIII, some simulations are shown. Section IX proposes an observed-based version of the same controller where only field existing measures are used. In the same section, we show also some simulations. And finally, Section X submits the conclusions and future research lines.

\section{BASICS ON DRILLSTRING DYNAMICS AND VIBRATIONS}

Standard rotatory drilling equipment, as shown in Figure 1 to depict what is commonly used by oil companies to extract gas and oil from the earth surface, uses a dill-bit (called bit) to crush the rock and make the hole in the ground. As the hole becomes deeper, some pipe sections (called drill pipes) are added, leaving the bit coupled at the bottom part of the set. These pipes, together with the drill bit, form the so-called drillstring. This drillstring is moved by means of a motor or system of motors in the surface. As it has been shown in the previous Section, operation of the drillstring looks just like that of a household electric drill, where a motor makes the bit rotate, and enough weight is applied to maintain the contact between the bit and the object to be drilled.

In order to make the study of a drilling system structure a bit more comprehensive, the following parts can be emphasized:

- Power System: A set of diesel and electric motors that provide the necessary energy to perform all the tasks.

- Supporting Structure: This is used to move the pipes in and out of the oil well, and so, to vary the weight applied during the process.

- The Rotatory System to make the system rotate. It is composed by:

- Swivel and Kelly: To connect the Supporting and Rotatory Systems.

- Rotatory Table: Also called turntable. It is a large disc-shaped inertia coupled to the drillstring that drives the rotating motion using power from electric motors.

- Drillstring: As shown before, it is a sequence of tubes that connect the rotatory table and the bit.

- Bit: The cutting device.

- Circulation System: It consists of a set of pipes and pumps which create a flow within the hole by drilling mud into it. This substance is aimed to lubricate and refrigerate the contact between the rock and the bit, and so to lift the rock cuttings from the drill bit to the surface.

A more exhaustive description of the rotatory system can be found, in [21] and [31] among other papers.

One of the main problems is the appearance of oscillatory behaviors (limit cycles), that cause a decreasing of the drilling performance from the viewpoints of different parameters (rate of penetration at the surface, rotational speed of the bit, ...) and so provoking the mechanical failure of the drillstring or the breakage of any of the elements [31]. 


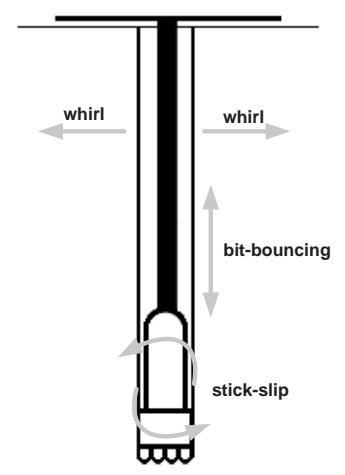

Fig. 2. Different types of vibrations in drillstring systems.

The vibrations appearing in the drillstring can be divided into 3 different categories [14] [10], (see Figure 2):

- Longitudinal vibrations are produced in a vertical direction from the drilling tower, causing rebounds of the bit at the bottom of the oil well, a phenomenon called bit-bouncing.

- Lateral vibrations are produced when the drillstring's mass center is displaced from the rotation axis, causing whirl-like movements and rebounds within the oil well walls, a phenomenon called whirling.

- Torsional vibrations are produced when the rotational velocities at the surface and the bottom of the drillstring are different, causing stick-slip movements.

Each oscillation phenomenon appears both at different times and different frequency ranges, and so, they can be studied separately. This work is focused on stick-slip vibrations.

The stick-slip oscillations are generally associated to typical dry friction profiles [19], i.e., when there is no movement, the friction torque (static friction) is larger than in non zero velocity cases (dynamic friction). The difference between those two magnitudes has been shown by many authors to be one of the most relevant variables that characterizes stick-slip oscillations [22].

\section{SySTEM MODELLING}

Multiple kind of models have been used in literature to describe drillstring systems (see for example [19] and [31]). The type and the complexity of the model to be used are closely related to the aim pursued (modelling, simulation, model for control, etc). However, lumped parameters models have been shown to be valid enough to properly describe the stick-slip oscillation phenomena and easy enough to make the study not too complex [10].

The problem of modelling stick-slip phenomenon in a drillstring by means of a lumped-parameter model has been studied from several points of view. Most of them consider the drillstring as a torsional pendulum with different degrees of freedom, for instance: [17], [20], [27], [32] propose single-degree-of-freedom models, [1], [5], [22], [24] 


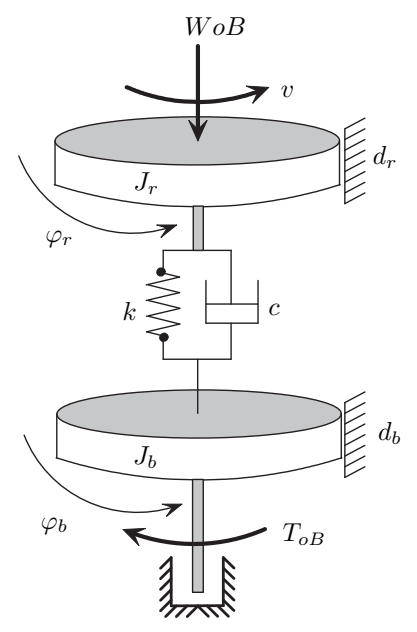

Fig. 3. Drillstring two-coupled masses model.

propose two-degree-of-freedom models including a linear controller, and [15], [29] present two-degree-of-freedom models for the mechanical part of the system plus the model for the rotary table electric motor system.

As it will be seen in subsequent sections, the bit-rock friction model is fundamental for properly reproducing stick-slip oscillations phenomenon. Many models have been proposed in literature, some of them summarized in [22]. The model used here (depicted in Figure 3) is a two-degree-of-freedom model with two inertial masses $J_{r}$ and $J_{b}$, locally damped by $d_{r}$ and $d_{b}$. The inertias are coupled with each other by an elastic shaft of stiffness $k$ and damping $c$. The variables $\varphi_{r}$ and $\varphi_{b}$ stand for the rotary and the bit angle. The rotary torque control signal $v$ used to regulate the rotary angular velocity $\dot{\varphi}_{r}$. The $\operatorname{ToB}$ (Torque on Bit) represents the total friction torque over the drill bit.

The model equations are the following:

$$
\begin{aligned}
& J_{r} \ddot{\varphi}_{r}+c\left(\dot{\varphi}_{r}-\dot{\varphi}_{b}\right)+k\left(\varphi_{r}-\varphi_{b}\right)+d_{r} \dot{\varphi}_{r}=v \\
& J_{b} \ddot{\varphi}_{b}+c\left(\dot{\varphi}_{b}-\dot{\varphi}_{r}\right)+k\left(\varphi_{b}-\varphi_{r}\right)+d_{b} \dot{\varphi}_{b}=-T o B
\end{aligned}
$$

In constants above, the sub-script ' $r$ ', and ' $b$ ' stands for rotary and bit, respectively.

A suitable model for $T o B$ is essential, because the reproduction of stick-slip vibrations will strongly depend on the particular choice of the model for $T o B$. This torque represents the combined effects of reactive torque on the bit and nonlinear frictional forces along the drillstring. In our case, the $T o B$ will be given by the product of $\mu\left(\dot{\varphi}_{b}, z\right)$, which describes the normalized (dimensionless) torsional bit-rock friction (different bit-rock friction models are presented in [22]), and the normal force $u$ called Weight on Bit $(W o B)$, i.e.

$$
T o B=\mu\left(\dot{\varphi}_{b}, z\right) \cdot u
$$

Several forms for $\mu\left(\dot{\varphi}_{b}, z\right)$ can be considered according the use of the model. Next, we describe the model for $T o B$ used for simulations and for validating the control law, then a simplified model is introduced for control analysis 
purposes.

\section{A. Model for simulations}

The state-space representation of the later model is the following:

$$
\begin{aligned}
\dot{x} & =A x+B v+H \mu(x, z) u \\
\dot{z} & =f(x, z)
\end{aligned}
$$

with

$$
A=\left(\begin{array}{ccc}
0 & 1 & -1 \\
\frac{-k}{J_{r}} & \frac{-\left(d_{r}+c\right)}{J_{r}} & \frac{c}{J_{r}} \\
\frac{k}{J_{b}} & \frac{c}{J_{b}} & \frac{-\left(c+d_{b}\right)}{J_{b}}
\end{array}\right), \quad B=\left(\begin{array}{c}
0 \\
\frac{1}{J_{r}} \\
0
\end{array}\right), \quad H=\left(\begin{array}{c}
0 \\
0 \\
\frac{-1}{J_{b}}
\end{array}\right)
$$

where the state $x=\left[x_{1} x_{2} x_{3}\right]^{T}$ is defined as follows:

$$
\begin{aligned}
& x_{1}=\varphi_{r}-\varphi_{b} \\
& x_{2}=\dot{\varphi}_{r} \\
& x_{3}=\dot{\varphi}_{b}
\end{aligned}
$$

In this description, the state $z \in R$ represents the internal friction state, and Equation (5) describes the friction dynamics. Various friction models have been shown to work properly to capture the typical friction phenomena (stiction, Stribeck effect, etc) which cause stick-slip oscillations ( [12] and [18]). One possible model for Equation (5) is the LuGre friction model [8]:

$$
\begin{aligned}
\dot{z} & =x_{3}-\sigma_{0} \frac{\left|x_{3}\right|}{g\left(x_{3}\right)} z \\
g\left(x_{3}\right) & =\mu_{C}+\left(\mu_{S}-\mu_{C}\right) e^{-\left(x_{3} / v_{s}\right)^{2}} \\
\mu(x, z) & =\sigma_{0} z+\sigma_{1} \dot{z}
\end{aligned}
$$

The function $g(v)$ it mainly affect the steady-state characteristics of the friction model. In steady-state, the model predict the following friction value, $\mu_{S S}\left(x_{3}\right)=g\left(x_{3}\right) \operatorname{sgn}\left(x_{3}\right)$. In this model, $\sigma_{0}, \sigma_{1}, v_{s}, \mu_{C}, \mu_{S}$ are positive constants characterizing the friction physical properties. Also note that the torsional linear friction at the drill bit side is already incorporated in the $A$ matrix of the representation (4).

\section{B. Model for control}

Note that the previous model for the $\mu(x, z)$ includes an additional friction dynamics, $z$ which is suited to describe motion at pre-sliding, and in particular to regularize the differential equation describing the system dynamics. An alternative is to use static description for $\mu(x)$ (maps without memory), which may be simple for control analysis. The different between both models, may not be too significant, as long as computation issues are strongly simplified. The model for control is then described by,

$$
\dot{x}=A x+B v+H \mu\left(x_{3}\right) u
$$


where here $\mu\left(x_{3}\right)$ is a static map between the bit rotational velocity $x_{3}=\dot{\varphi}_{b}$ and the normalized friction parameter $\mu$, i.e. the steady-state form of the model (7), or any suitable approximation like the one shown in Figure 12.

\section{Rotational Velocity Regulation LoOP}

The first task to confront is designing a proper control law for motor torque $v$. This signal will be aimed mainly at regulating the rotational velocity of the rotatory table $\dot{\varphi}_{r}$ to a certain desired value $\omega_{d}$ (a typical value for $\omega_{d}$ is $5 \mathrm{rad} / \mathrm{s}$ ). As we have shown in Section I, many architectures have been proposed for that purpose, from classic PID structures to a linear $H_{\infty}$ robust control, although oil well drillstrings usually operate with reduced-order simple control laws.

\section{A. Rotary table velocity control loop}

In this work, the structure of the velocity controller is inspired by the one presented in [10], as shown by:

$$
v=\left[k_{1}+\frac{k_{2}}{s}\right]\left(\omega_{d}-\dot{\varphi}_{r}\right)-k_{3}\left(\dot{\varphi}_{r}-\dot{\varphi}_{b}\right)
$$

or equivalent

$$
\begin{aligned}
v & =k_{1}\left(\omega_{d}-x_{2}\right)+k_{2} x_{4}-k_{3}\left(x_{2}-x_{3}\right) \\
\dot{x}_{4} & =\left(\omega_{d}-x_{2}\right)
\end{aligned}
$$

then the closed-loop equations take the form for the model for simulation,

$$
\begin{aligned}
\dot{x} & =A_{c l} x+B_{c l} \omega_{d}+H_{c l} \mu(x, z) u \\
\dot{z} & =f(x, z)
\end{aligned}
$$

and the following one for the model for control analysis,

$$
\dot{x}=A_{c l} x+B_{c l} \omega_{d}+H_{c l} \mu\left(x_{3}\right) u
$$

with the obvious observation that $x$ is now of dimension four (due to the introduction of an integral term in the rotary table control), i.e. $x=\left[x_{1}, x_{2}, x_{3}, x_{4}\right]$, and with the $A_{c l}, B_{c l}$ and $H_{c l}$ given as:

$$
A_{c l}=\left(\begin{array}{cccc}
0 & 1 & -1 & 0 \\
\frac{-k}{J_{r}} & \frac{-\left(d_{r}+c+k_{1}+k_{3}\right)}{J_{r}} & \frac{\left(c+k_{3}\right)}{J_{r}} & \frac{k_{2}}{J_{r}} \\
\frac{k}{J_{b}} & \frac{c}{J_{b}} & \frac{-\left(c+d_{b}\right)}{J_{b}} & 0 \\
0 & -1 & 0 & 0
\end{array}\right), \quad B_{c l}=\left(\begin{array}{c}
0 \\
\frac{k_{1}}{J_{r}} \\
0 \\
1
\end{array}\right), \quad H_{c l}=\left(\begin{array}{c}
0 \\
0 \\
\frac{-1}{J_{b}} \\
0
\end{array}\right)
$$

The steady-state value of $x$, considering $\mu^{*}=\mu\left(x_{3}^{*}\right)$, is:

$$
\begin{aligned}
x_{1}^{*} & =\frac{u_{0} \mu^{*}+d_{b} \omega_{d}}{k} \\
x_{2}^{*} & =\omega_{d} \\
x_{3}^{*} & =\omega_{d} \\
x_{4}^{*} & =\frac{\left(d_{b}+d_{r}\right) \omega_{d}+\mu^{*} u_{0}}{k_{2}}
\end{aligned}
$$




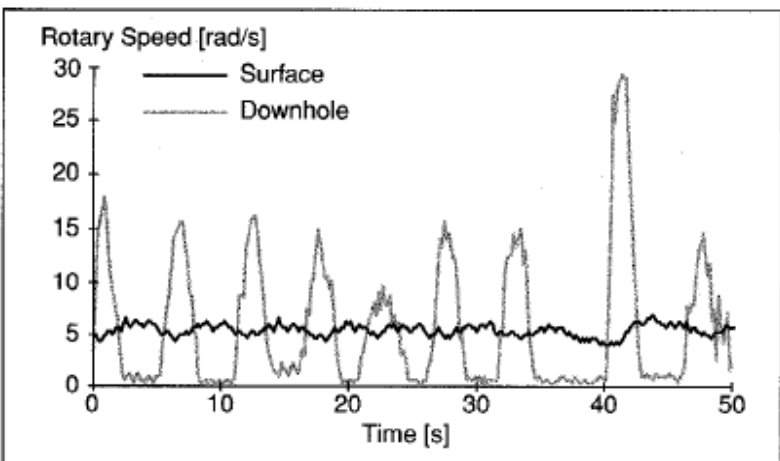

a)

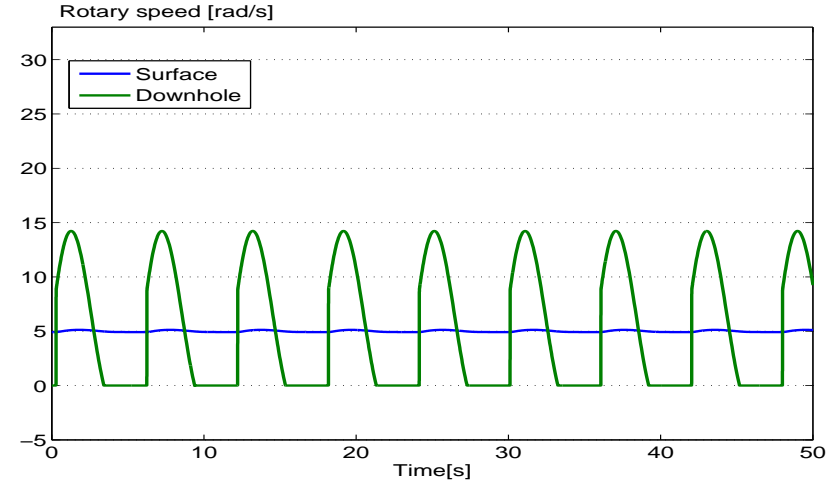

b)

Fig. 4. Stick-slip measured in the field (a) (taken from [29]) and simulation profiles obtained with the closed-loop model (b).

Therefore, rotatory table and bit velocities stabilize at the desired rotational velocity, revealing a proper feature in our feedback control law.

There are many possible ways of adjusting the gains $k_{1}, k_{2}$ and $k_{3}$. One possible option is to use a classic two-time-scales separation method to accomplish this (see, for example [9]). Further details are given in Appendix A.

\section{B. Closed-loop system model validation}

Once the control torque has been designed and tuned, the global performance of the model in terms of reproducing the behavior of real drilling equipment, particularly the ability of reproducing stick-slip oscillations, must be validated.

In Figure 4, a comparison between stick- slip oscillations measured in the field (Figure obtained with permission from [29]) and the ones produced by simulating the closed-loop system (10)-(11) can be seen. Note that, although the model does not include the lateral and vertical motion dynamics, it is able to reproduce trajectories that are qualitatively close to the ones reported from field data in terms on frequency, amplitude, and shape. Irregularities in the oscillation amplitudes of the field measures are probably due to the vertical bouncing oscillations not included in our model.

\section{PRELIMINARY ANALYSIS OF STICK-SLIP OSCILLATIONS BEHAVIOR}

With regards to the oscillatory behavior of drillstring system, it is assumed that, depending on certain working parameters like the instantaneous value of $W_{o} B$, the rotatory speed of the set, the velocity control gains values, and so forth, the drillstring system can enter into oscillation.

There will be cases in which the controller can suppress such oscillation, and so, the system recovers its nominal working regime, and other cases in which the system enters into sustained oscillation. This behavior is observed in real drillstring equipment, as seen in Figure 4. 
A particular feature of the model presented here (for simulations) is that it succeeds in reproducing such behavior, as it can be seen in Figure 5, where the $W o B$ parameter is perturbed away from its nominal operation value; in the left (upper) corner of Figure 5, the $W o B$ changes from $40000 N$ to $45000 N$ and the controller is still able to correctly regulate the rotatory speed, whereas in the left (lower) corner of Figure 5 the $W o B$ is perturbed enough (from $40000 N$ to $50000 N$ ) so that the velocity controller cannot compensate the perturbation and the system enters into stick-slip oscillation.
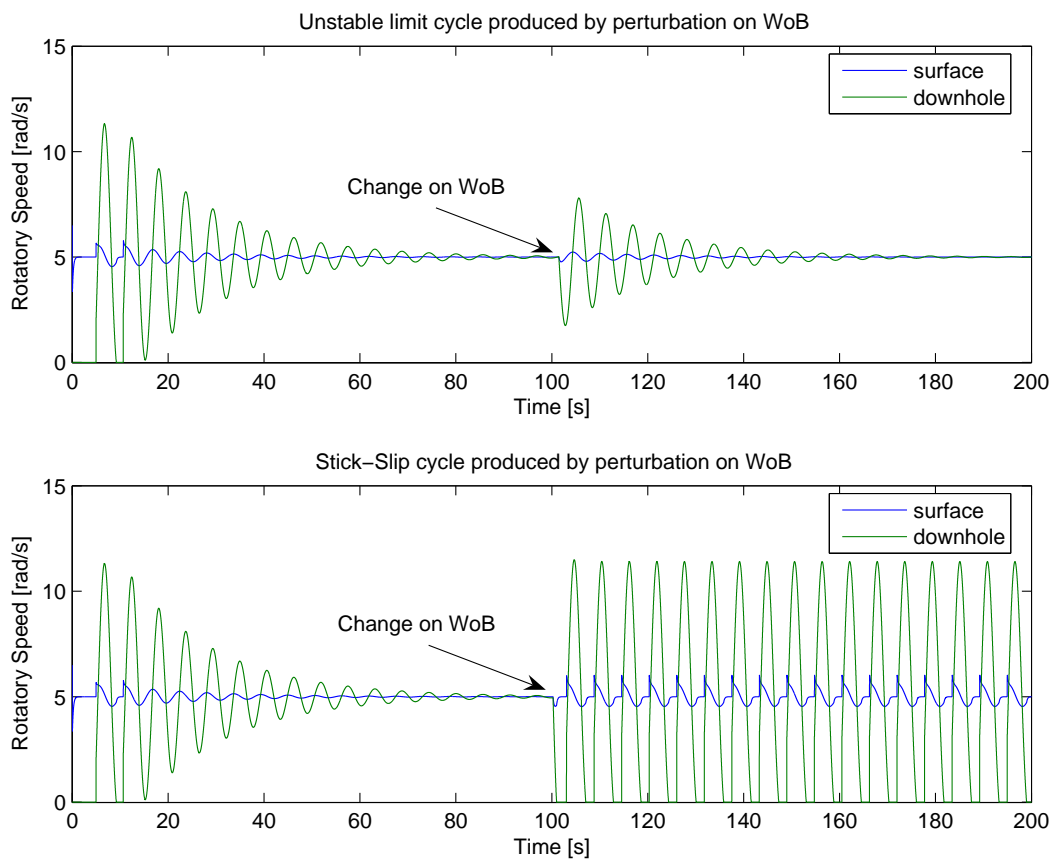

Fig. 5. Different behaviors obtained by perturbing $W o B$. Figures show the time-profiles of the table and bit speed.

\section{A. Intuitive behaviour}

Although the system at hand is more complex, the following explanation of the system behaviour gives some intuition on how the system may operated. The system trajectories can intuited to behave like the ones in Figure 6 . From this figure, we expect that the behavior of a nonlinear system will display a local stable region with a stable limit set outside this local attraction domain (some more formal statements will be given latter). This is the pattern display with our system when $u$ is assumed to be constant. Indeed, the system behavior is even more complex when $u$ can take on different values. For instance, a saddle-node bifurcation of periodic orbits may be exhibited if the value of $u$ is decreased enough. In that case, two limit cycles emerge at the bifurcation point, one of them being stable while the other is unstable. As the system has an equilibrium point, its local stability is not affected by the emergence of such limit cycles. Furthermore, for values of $u$ smaller than the one corresponding to the bifurcation point, limit cycles disappear and the equilibrium becomes globally stable. 


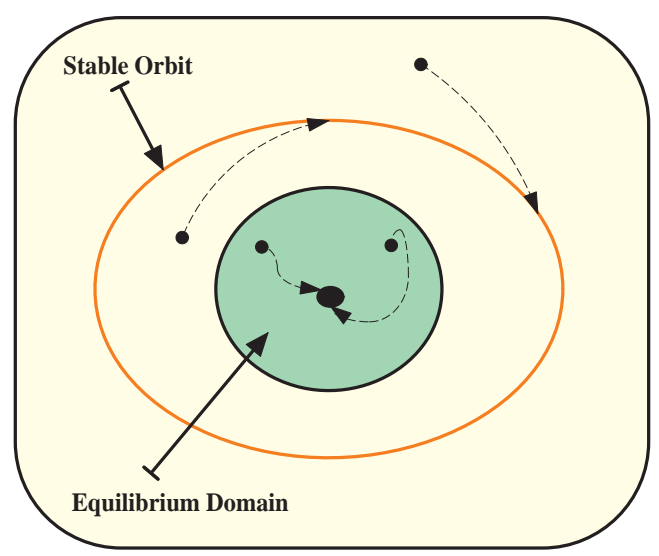

Fig. 6. Figure presents the general idea of a local equilibrium encircled by a stable limit cycle. When the perturbation is not too large system trajectories return to equilibrium. When the perturbation is large enough, the trajectory converge to a limit cycle.

\section{STICK-SLIP OSCILLATIONS PREDICTION BASED ON DF-ANALYSIS}

The oscillation pattern shown by Figure 4 reflects two main characteristics: first oscillations will present a bias term, and second they are dominated by a main harmonic with a relatively well-defined period. Therefore, the SBDF (Sinusoid plus bias describing function) method can then be used as a first approximation to predict possible limit cycle and to study its stability. To further simplify the computation of the SBDF corresponding to the nonlinear dynamic friction map (7), we would rather use a simpler approximation of the resulting steady-state characteristic of (7), see Figure 7. This approximation is valid because the friction dynamics are much faster than the ones of the drillstring mechanism.

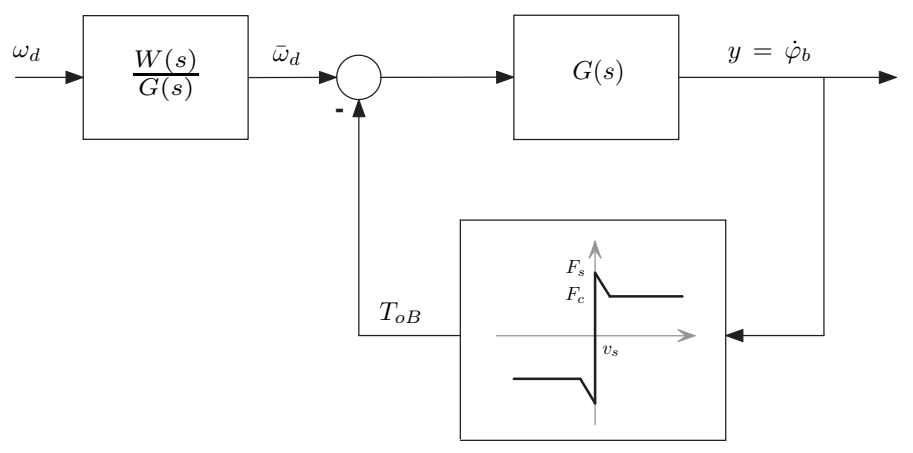

Fig. 7. System block diagram and static friction torque.

The setup for this study is shown in Figure 7. $G(s)$ is the linear map from $G(s):\left(\bar{\omega}_{d}-T o B\right) \rightarrow y=\dot{\varphi}_{b}$, and $\bar{\omega}_{d}$ is the resulting bias due to the constant reference $\omega_{d}$.

Applying harmonic balance, the necessary condition to produce maintained oscillations in the system is:

$$
G\left(j \omega_{0}\right)=\frac{-1}{N_{1}\left(A_{0}, \omega_{0}, y_{0}\right)}
$$




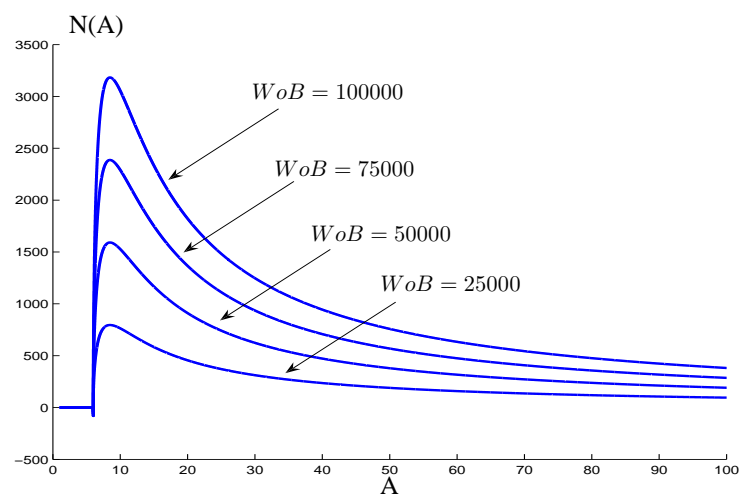

a)

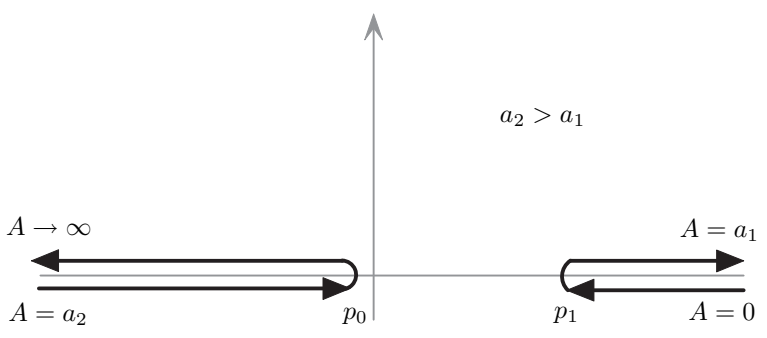

b)

Fig. 8. Describing function $N(A)$ (a), and evolution of $\frac{-1}{N(A)}$ (b). $\left|p_{0}\right|$ is two or three magnitude orders lower than $\left|p_{1}\right|$, depending on value of $W o B$.

$$
y_{0}\left[1+G(0) N_{0}\left(A_{0}, y_{0}\right)\right]=W(0) \omega_{d}
$$

$A_{0}$ and $\omega_{0}$ are the particular values satisfying both equalities. They represent the amplitude and frequency of the predicted oscillations, if any. $y_{0}$ is the output bias, and $N_{1}$ and $N_{0}$ are given in the Appendix B.

Note that $G(j \omega)$ depends on the control parameters, hence the assigned bandwidth $\omega_{n}$.

Since the observed oscillations are clearly asymmetrical, the SBDF is used to compute $N_{1}\left(A, \omega, y_{0}\right)$, see [4]. $N_{1}\left(A, y_{0}\right)$ will have only a real part because the friction torque characteristic has an odd symmetry.

Note that the frequency locus of $G\left(j \omega_{0}\right)$ can only cross the real axis at one point, then $\omega_{0}$ can be uniquely computed from $\operatorname{Im}\left\{G\left(j \omega_{0}\right)\right\}=0, \forall \omega_{0} \neq 0, \quad\left|\omega_{0}\right|<\infty$, and hence it does not depend on the other two unknown variables $A_{0}$ and $y_{0}$. The introduction of a integral action in the velocity control results in $G(0)=0$, and $W(0)=1$. Therefore, the output bias can be straightforwardly computed from equation (18). Yielding

$$
y_{0}=\frac{W(0)}{\left[1+G(0) N_{0}\left(A_{0}, y_{0}\right)\right]} \omega_{d}=\omega_{d}
$$

With $y_{0}=\omega_{d}$ known and independent of frequency and amplitude, the prediction of the limit cycle and its associated amplitude $A_{0}$, can be obtained only from equation (17), i.e.

$$
\mathcal{R} e\left\{G\left(j \omega_{0}\right)\right\}=-\frac{1}{N\left(A_{0}\right)}
$$

with $N\left(A_{0}\right)=N_{1}\left(A, \omega_{d}\right)$.

Figure 8 shows how $\frac{-1}{N(A)}$ changes as a function of $A$. The main feature of this amplitude locus, pertinent to oscillation predictions, is the location of points $p_{0}$ and $p_{1}$. These points derive from the maximum and minimum values of $N(A)$. In particular, it is interesting to notice that these points have the following property:

$$
\left|p_{0}\right| \sim \frac{1}{W o B} \quad \text { and } \quad\left|p_{1}\right| \sim \frac{1}{W_{o B}}
$$

Then, low values of $W o B$ make these two points larger in magnitude (its sign remains unchanged), reducing the possibility to intersect the Nyquist of $G(j \omega)$. Inversely, when the weight on bit force increases, the probability of 


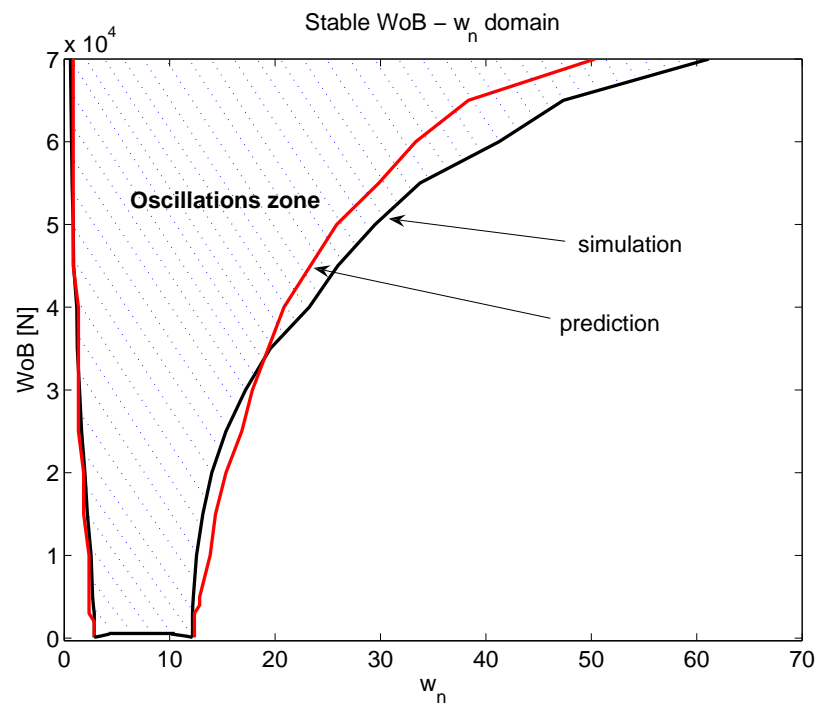

Fig. 9. Oscillations frequency domain. Border obtained by simulations and border predicted from the SBDF analysis.

oscillation is higher.

It is also interesting to note, that for almost every realistic choice of $\omega_{n}$, two sets of limit cycles will be predicted; one stable set and another unstable. The stable set arises at amplitudes $A=A_{s}$, whereas the unstable one occurs for $A=A_{u}$. In all cases $A_{s}>A_{u}$. This means that for all parameter combinations $\left(W o B-\omega_{n}\right)$ where an intersection of $G(j \omega)$ and $-1 / N(A)$ takes place, there exists a local (attractive) stable domain delimited by $A_{u}$. This property is further exploited in the proposed D-OSKIL mechanism.

It is also of interest to compute the set of possible combinations between system bandwidth and weight on bit force for which stable limit cycles are predicted. Figure 9 shows the area in the $\left(W o B-\omega_{n}\right)$-plane giving rise to possible stable oscillations. This analysis allow us to determine the range of proper values of controller closed-loop bandwidth to avoid oscillations as a function of the operating $W o B$. As the figure shows, and on the basis of this analysis, oscillations may be eliminated either by changing $\omega_{n}$, and/or by reducing the $W_{o} B$ magnitude.

One possible control strategy could be then to modify the rotary table bandwidth to reduce the possibility of entering into oscillation. From Figure 9, we can see that suitable choices for $\omega_{n}$ are either small or large values. Small values are not suited because they yield to poor performance, and large values are limited by noise. Typical values for $\omega_{n}$ are in the range $[20,30] \mathrm{rad} / \mathrm{s}$.

Another alternative will be to keep the value of $W o B$ below the oscillation zone. However, this will have two drawbacks: first, high-performance drilling operations require magnitudes for $W o B$ larger than the ones indicated by the oscillation limits, and second, this strategy will require a precise knowledge of this map, which in addition will vary according to drilling depth. Keeping $W o B$ low, will thus be a too conservative strategy, which may cause exploitation time to increase.

High-performance drilling operation takes place in a region in the plane $\left(W o B-\omega_{n}\right)$, where potential oscillations 
may occur. Therefore, in this paper we account for this particularity assuming that the nominal system operation parameters are taken within this zone. The design of the D-OSKIL mechanisms presented next, will be performed in light of the following two observations:

- oscillation can be eliminated by decreasing the Weight on Bit force, and

- if $W o B$ is reduced from its nominal value, then after the oscillation has been eliminated, the value of $W o B$ needs to be brought back to its nominal value again to restart an efficient drilling operation.

\section{THE D-OSKIL MECHANISM DESIGN}

This section presents the design of the D-OSKIL mechanism, which stands for Drilling OScillation KILler. First we introduce some intuitive ideas completing the understanding on the system behaviors gained in previous sections. Then we present the mathematical form for this algorithm and the different steps involved in their stability properties.

\section{A. Basic Ideas}

Let us first assume that the normal force $u$ (i.e. the weight on Bit $W_{o B}$ ), is given as the sum of the nominal weight of the drillstring $\left(u_{0}\right)$ and the D-OSKIL control signal $(\tilde{u})$ :

$$
u=u_{0}+\tilde{u}
$$

Figure 10 is a schematic description of what we expected to be the behavior of the system. Although it is not formal, we may think of this Figure as a bifurcation diagram, where in the horizontal axis we have the "bifurcation parameter" $u$, and in the vertical axis a measure of the oscillations (i.e. the Euclidian norm of the error vector, to be defined later). As previously discussed, $u_{0}$ is here the nominal value for $u$ of an efficient drilling operation. The upper solid line describes a possible family of stable oscillations, whereas the lower dotted line is a family of unstable ones. The lines with arrows describe possible trajectories of the closed-loop system parameterized as a function of $u$. These trajectories are extrapolated from the a priori behavior of the drillstring system.

There are two interesting cases to be analyzed:

a) Nominal behavior: Assume that $u=u_{0}$, and the system trajectories are at equilibrium. Then if a perturbation arrives:

- Either the perturbation is not large enough to divert the system trajectories from their local attraction domain (a curve in figure). Therefore the velocity controller succeeds in regulating the trajectories back to their equilibrium. Or,

- The perturbation is large enough to divert the system from equilibrium domain. In this case (b curves in figure), the trajectories are attracted by the corresponding stable limit cycle and the system enters into sustained oscillation. 


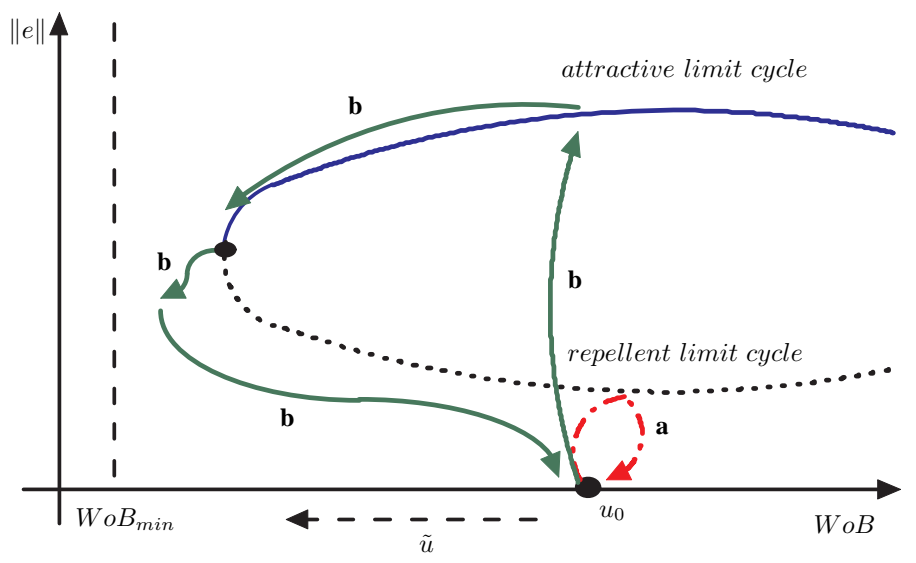

Fig. 10. Possible drillstring system trajectories.

b) Behavior beyond nominal operations: To suppress such oscillations, the $u=W o B$ must be reduced by means of the control signal $\tilde{u}$, until its trajectory reaches the bifurcation point, and the local controller is able to return the system trajectories to the equilibrium. Thereafter, the nominal value of the $W o B$ must be recovered in a proper slow manner, to continue with the drilling task, i.e. $u \rightarrow u_{0}$. Significantly enough, the variation of $\tilde{u}$ should be restricted to a valid domain, and in particular restricted to a positive values other than zero. Without this restriction, it is clear that drilling may not be efficient, or it will be impractical.

The general structure of the variation law for $u$ (or equivalent for $\tilde{u}$ ), will be here of the form:

$$
\dot{\tilde{u}}=\mathcal{P}_{-u_{0}}^{0}\{-\sigma \tilde{u}+\Phi(\cdot)\}
$$

where $\sigma>0$, can be understood as a time-constant of the controller, and $\mathcal{P}_{-u_{0}}^{0}$ is a projector operator ensuring that solutions of the above equation makes $\tilde{u}$ stay in the range $\left(-u_{0}, 0\right]$, and $\Phi(\cdot)$ is a nonlinear function which must be designed to ensure system stability, that is:

$$
\dot{\varphi}_{r} \rightarrow \omega_{d} \quad \text { and } \quad \tilde{u} \rightarrow 0
$$

In order to make the presentation simpler, we will trop the explicit use of the projection operation $\mathcal{P}$ in the following section. However, the reader should keep in mind that $\tilde{u}$ is a bounded signal in the prescribed range.

With this in mind, the complete closed-loop equations are:

$$
\begin{aligned}
\dot{x} & =A_{c l} x+B_{c l} \omega_{d}+H_{c l} \mu\left(x_{3}\right)\left(u_{0}+\tilde{u}\right) \\
\dot{\tilde{u}} & =-\sigma \tilde{u}+\Phi(\cdot)
\end{aligned}
$$

where matrices $A_{c l}, B_{c l}, H_{c l}$ have been defined previously. 


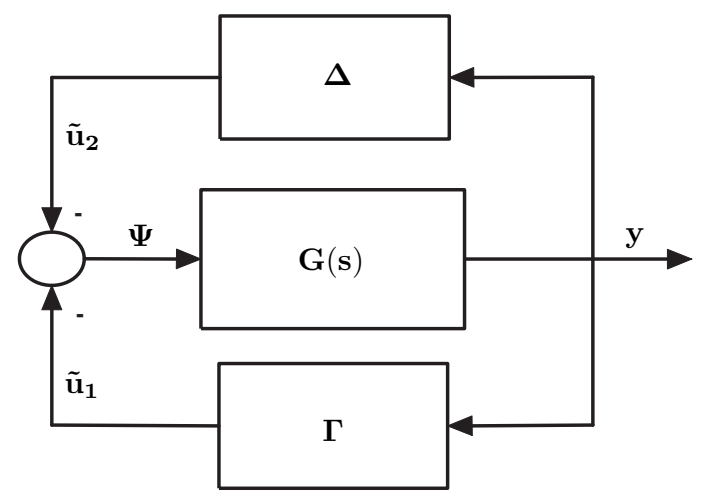

Fig. 11. Error system block diagram.

\section{B. Error equations}

Error equations can be obtained by applying the change of coordinates $e=x-x^{*}$, and considering the steady-state values $\mu\left(e_{3}\right)_{s s}=\mu^{*}$ and $\tilde{u}_{s s}=0$. This yields ${ }^{2}$ :

$$
\begin{aligned}
\dot{e} & =A_{c l} e+H_{c l}\left[\mu(y) \tilde{u}+\tilde{\mu}(y) u_{0}\right] \\
\dot{\tilde{u}} & =-\sigma \tilde{u}+\Phi(y) \\
y & =C e=e_{3}
\end{aligned}
$$

where the term $\tilde{\mu}(y)$ is defined as follows:

$$
\tilde{\mu}(y)=\mu(y)-\mu^{*}
$$

and we assume that the update rule for $\tilde{u}$ is designed on the basis of the output $y$. The error system can be described by the block diagram in Figure 11, with the following definitions:

$$
\begin{aligned}
& G(s): \quad \Psi \mapsto y \\
& \Gamma \quad: \quad y \mapsto \tilde{u}_{1}=\mu(y) \tilde{u} \\
& \Delta \quad: \quad y \mapsto \tilde{u}_{2}=\tilde{\mu}(y) u_{0}
\end{aligned}
$$

where $\Psi=-\left(\tilde{u}_{1}+\tilde{u}_{2}\right)$.

\section{Error equation properties}

1) PR condition on $G(s)$ : The map $G(s)$ is:

$$
G(s)=-C\left(s I-A_{c l}\right) H_{c l}
$$

${ }^{2}$ With an abuse of notation, we will use $\mu(y)$ to denote the expression of $\mu\left(x_{3}\right)$, in the shift coordinate $y+\omega_{d}$, i.e. $\mu\left(x_{3}\right)=\mu\left(y+\omega_{d}\right)=\mu(y)$. Note that at $y=0$, we have $\mu(y=0)=\mu\left(\omega_{d}\right)=\mu^{*}$. 


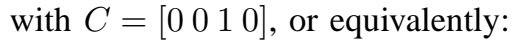

$$
G(s)=\frac{s\left(a_{2} s^{2}+a_{1} s+a_{0}\right)}{b_{4} s^{4}+b_{3} s^{3}+b_{2} s^{2}+b_{1} s+b_{0}}
$$

The error equation and updating rule of $\tilde{u}$ have been designed in such a way that the resulting map $G(s)$ has relative degree one. This condition is necessary to obtain PR and SPR functions. The verification that $G(s)$ is PR can be done directly on the triplets of matrices $\left(A_{c l}, H_{c l}, C\right)$ as demonstrated in [30] for SPR function. Adapting this result to PR functions and using our notation at hand, results in the following relaxed conditions: consider the transfer function $G(s)=-C\left(s I-A_{c l}\right) H_{c l}$. $G(s)$ is PR if and only if: 1) $\left.C A_{c l} H_{c l}>0,2\right) A_{c l}$ is stable, 3) the matrix $A_{c l}\left(I-\left(1 / C A_{c l} H_{c l}\right) A_{c l} H_{c l} C\right) A_{c l}$ has no eigenvalues on the open negative real axis $(-\infty, 0)$.

The first condition is easy to compute in terms of model parameters. This gives $C A_{c l} H_{c l}=\left(c+d_{b}\right) / J_{b}^{2}>0$, and always holds from the physics of the system. The second condition is also verified since $A_{c l}$ is designed to be stable. The last condition is more involved, but it can be easily checked numerically. For typical values of drillstring system model parameters and $v$-control gains considered in this paper, it is possible to show that condition 3) holds. Note also that by continuity of the eigenvalues with respect to the matrices parameters, there will exists a certain degree of robustness of this condition with respect the model uncertainly.

Consequently we have that $G(s)$ is a Positive Real (PR) function, and hence from the Kalman-Yacubovich-Popov Lemma [16], the following property holds: $\exists P=P^{T}>0, Q=L^{T} L \geq 0$ such that:

$$
\begin{aligned}
A_{c l}^{T} P+P A_{c l} & =-Q=-L^{T} L \leq 0 \\
P H_{c l} & =-C
\end{aligned}
$$

Therefore, as a consequence we have the following two properties for the linear map $G(s)$ :

- $G(s)$ is a passive relative to $V(e)=e^{T} P e$, and

- $G(s)$ has a finite $L_{2}$-gain: $\gamma_{2}(G)=\sup _{\omega}|G(j \omega)|<\infty$

2) Boundedness of signal $\Psi(t)$ : From the definitions of $\tilde{u}_{1}$ and $\tilde{u}_{2}$ in Equations (27) and (28), together with the assumption that the adaptation mechanism yields values in the range $\tilde{u} \in\left(-u_{0}, 0\right]$, it follows that both signals, $\tilde{u}_{1}$ and $\tilde{u}_{2}$, are bounded, that is:

$$
\begin{aligned}
& \left\|\tilde{u}_{1}\right\|_{\infty}=\sup _{t \geq 0}\left|\tilde{u}_{1}\right| \leq u_{0}<\infty, \\
& \left\|\tilde{u}_{2}\right\|_{\infty}=\sup _{t \geq 0}\left|\tilde{u}_{2}\right| \leq 2 \cdot u_{0}<\infty .
\end{aligned}
$$

Hence $\|\Psi(t)\|_{\infty} \leq 3 \cdot u_{0}$.

3) Boundedness of the output $y(t)$ : Since $G(s): \Psi \mapsto y$ is a lineal stable map, the output signal $y$ is also bounded, i.e.

$$
\Psi \in L_{\infty} \Rightarrow y \in L_{\infty}
$$

4) Sector condition on $\Delta$ : With regard to Figure 11, the output of the map $\Delta$ can be seen as a disturbance acting on the closed loop system resulting from the operators $G(s)$ in feedback connection with nonlinear operator $\Gamma$. 


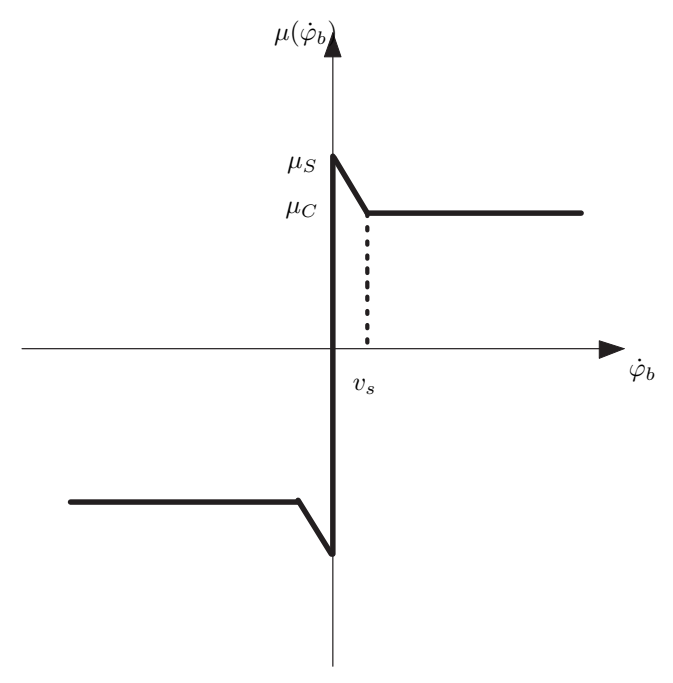

a)

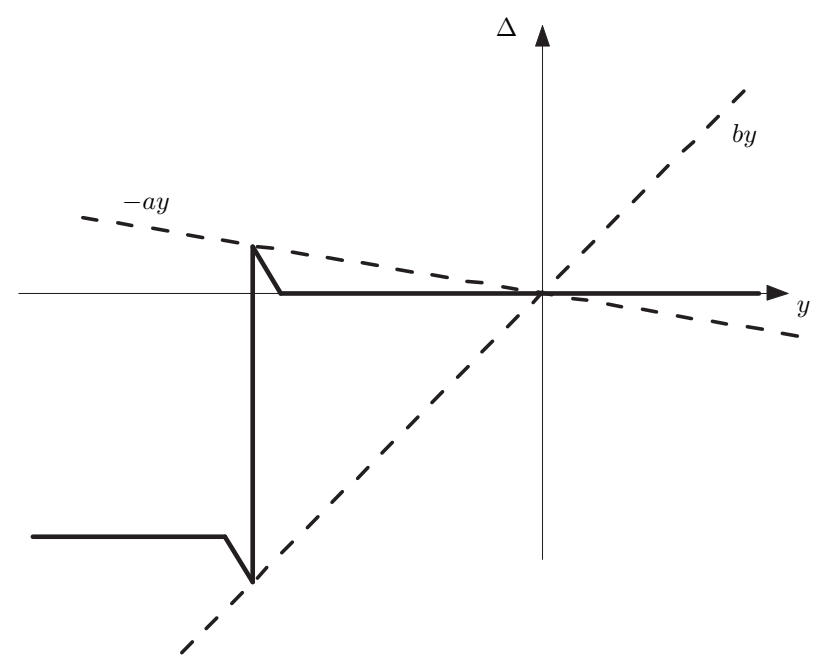

b)

Fig. 12. Normalized friction function (a). $\Delta(y)$ lies at the interval $[a, b]$ (b).

Figure 12-(a) shows the memoryless friction map used for this study. Note that as the steady-state rotatory bit speed $\left(\omega_{d}\right)$ is in general much larger than the Stribeck velocity $v_{s}$, we can then assume that $\mu^{*}=\mu_{C}$. Therefore, taking into account that $y=e_{3}=\dot{\varphi}_{b}-\omega_{d}$, the output of block $\Delta$ will have the profile shown in Figure 12-(b). This operator belongs to the cone sector $[a, b]$ as displayed in the same Figure (see [33] for further discussion on sector definitions). Formally this is stated as follows.

The nonlinear operator $\Delta(y)$ belongs to the sector $[a, b]$ if the following holds true:

- $\Delta(0)=0$

- $a \leq \frac{\Delta(y)}{y} \leq b, \forall y \geq 0$, or equivalently,

- $a y^{2} \leq y \Delta(y) \leq b y^{2}, \forall y \in \Re$

In our case, the values for $a$ and $b$ are:

$$
\begin{aligned}
a & =-\frac{\mu_{S}-\mu_{C}}{\omega_{d}} u_{0} \\
b & =\frac{\mu_{S}+\mu_{C}}{\omega_{d}} u_{0}
\end{aligned}
$$

and consequently, the map $\Delta$ has also finite $L_{2}$-gain, which is bounded by:

$$
\gamma_{2}(\Delta) \leq \max [|a|,|b|]
$$

5) Block transformation: As it can be seen in Figure 12-(b), the map $\Delta$ is almost passive, since almost the whole diagram is within the first and third quadrants. This characteristic is generic, as the difference between break-away and Coulomb friction levels is generally small ( $a$ is small when compared to $b$ ). 
Figure 13 shows a possible block transformation, where the following new operators, $\Delta^{*}$, and $\Gamma^{*}$ are defined:

$$
\begin{aligned}
\Delta^{*} & : \quad y \mapsto\left(\tilde{u}_{2}+\varepsilon y\right) \\
\Gamma^{*} & : \quad y \mapsto\left(\tilde{u}_{1}-\varepsilon y\right)
\end{aligned}
$$

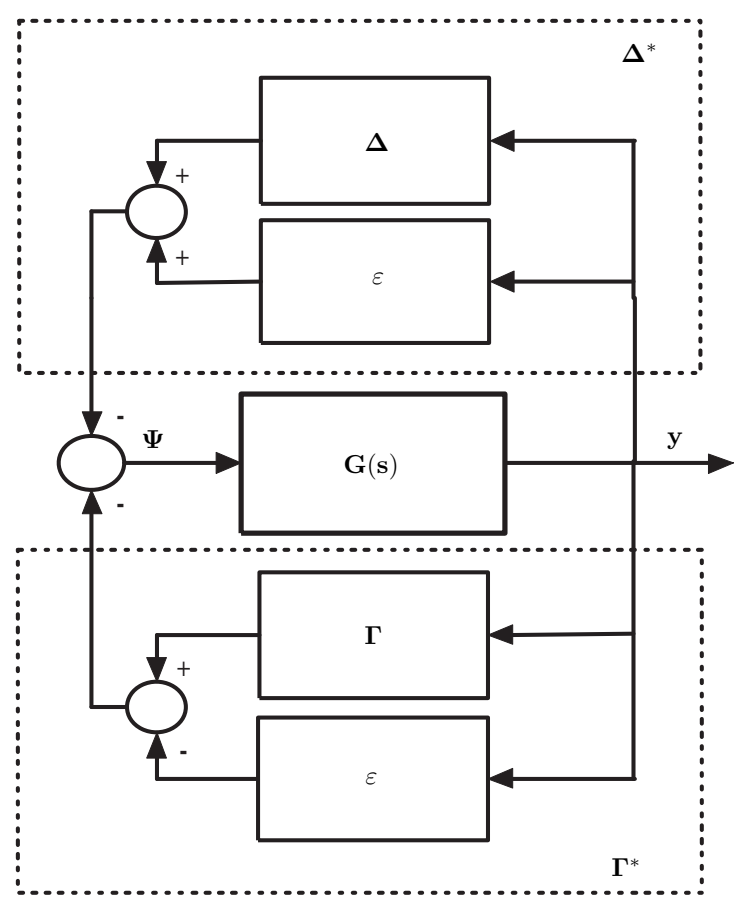

Fig. 13. Modified Block Diagram.

With this transformation, it can be easily proved that the map $\Delta^{*}$ is passive if the value of $\varepsilon$ is taken such that $\varepsilon=|a|$, as can be seen in Figure 14 .

$$
\varepsilon=|a| \Rightarrow \int_{0}^{t} y\left(\tilde{u}_{2}+\varepsilon y\right) d t \geq 0
$$

With this new feedback configuration, the problem of designing a stable update law for $\tilde{u}(t)$ is equivalent to finding a function $\Phi(y)$, and parameter conditions, such that the transformed operator $\Gamma^{*}$ defines a passive map. This design strategy results from well known properties of feedback interconnected passive systems.

The next subsection uses such a result to demonstrate the stability properties of one possible candidate update rule.

\section{D-OSKIL updating law}

Under the premise that the complete form of the update law should also include a suited projection operator ensuring that the variation of $\tilde{u}$ is limited to the admissible parameters range, the following updating rule will be analyzed. 


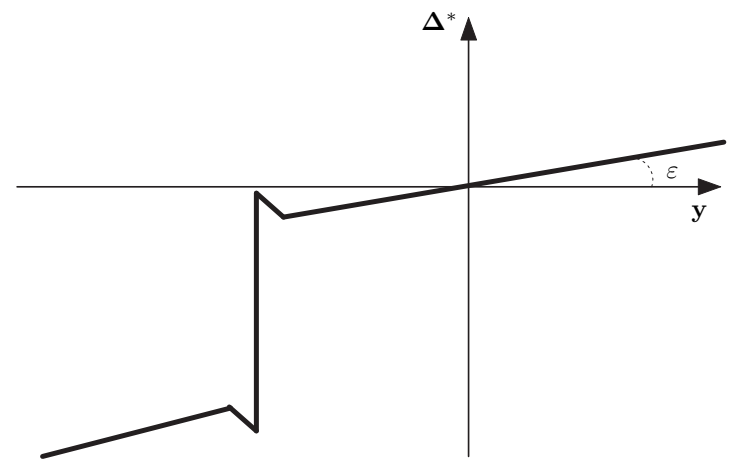

Fig. 14. Profile of map $\Delta^{*}$.

Let us consider the nonlinear function $\Phi(y)$ :

$$
\Phi(y)=\lambda y \operatorname{sgn}(\mu(y)) \quad \lambda \geq 0
$$

Note that this choice is conditioned by the ability of computing the sign of $\mu(y)$. Considering the form of the friction model for this study, the sign of $\mu(y)=\mu\left(\dot{\varphi}_{b}-\omega_{d}\right)$, can be computed if $\dot{\varphi}_{b}$ can be measured, or at least, observed as shown in simulations later on. We proceed according to this hypothesis in what follows.

\section{E. Stability analysis}

Lemma 1: Let $\rho>0$ be an arbitrarily positive constant, and $\lambda, \sigma$ be such that the following design inequality holds,

$$
\frac{\lambda}{\sigma} \geq\left(\frac{\mu_{S}}{\mu_{C}}-1\right) \frac{u_{0}}{\omega_{d}}+\frac{\sigma}{\mu_{C}} \rho
$$

where $\frac{\mu_{S}}{\mu_{C}} \geq 1$. Then map $\Gamma^{*}: y \mapsto(\mu(y) \tilde{u}-\varepsilon y)$ is strictly input passive, i.e.

$$
I=\int_{0}^{t}(\mu(y) \tilde{u}-\varepsilon y) y \geq \rho \int_{0}^{t} y^{2}-\beta_{0}
$$

with $\beta_{0}=\frac{y_{\max }}{\sigma} 2 u_{0}>0$.

Proof: Let $I$ define the integral of the input-output product of the operator $\Gamma^{*}$, i.e.

$$
I=\int_{0}^{t}(\mu(y) \tilde{u}-\varepsilon y) y=\int_{0}^{t} \mu(y) \tilde{u} y-\int_{0}^{t} \varepsilon y^{2}
$$

Substituting $\tilde{u}$ from Equation (23) in the above expression gives,

$$
I=\frac{1}{\sigma} \int_{0}^{t} \mu(y) \Phi(y) y-\frac{1}{\sigma} \int_{0}^{t} \mu(y) y \dot{\tilde{u}}-\int_{0}^{t} \varepsilon y^{2}
$$

From sections VII-C.2, and VII-C.3, signals $y$ and $\mu(y)$ have been shown to be bounded. Let note these bounds as: $|y|<y_{\max },|\mu(y)|<1$. Therefore, 


$$
I \geq \frac{1}{\sigma} \int_{0}^{t} \mu(y) y \Phi(y)-\int_{0}^{t} \varepsilon y^{2}-\frac{y_{\max }}{\sigma}\left|\int_{0}^{t} \dot{\tilde{u}}\right|
$$

Taking now $\Phi(y)=\lambda y \operatorname{sgn}(\mu(y))$, gives

$$
\begin{aligned}
I & \geq \int_{0}^{t}\left(\frac{\lambda}{\sigma}|\mu(y)|-\varepsilon\right) y^{2}-\frac{y_{\max }}{\sigma}|\tilde{u}(t)-\tilde{u}(0)| \\
& \geq \int_{0}^{t}\left(\frac{\lambda}{\sigma}|\mu(y)|-\varepsilon\right) y^{2}-\frac{y_{\max }}{\sigma} 2 u_{0} \\
& \geq \int_{0}^{t}\left(\frac{\lambda}{\sigma} \mu_{C}-|a|\right) y^{2}-\beta_{0}
\end{aligned}
$$

where the last inequality is obtained by using the lower bound on $|\mu(y)|$, i.e., $|\mu(y)| \geq \mu_{C}$, the definition of $\varepsilon=|a|$ (with $a$ as given in Equation (36)) and the constant $\beta_{0}=\frac{y_{\max }}{\sigma} 2 u_{0}$. Finally, introducing the condition (41) in the above expression, gives the following lower bound on $I$ :

$$
I \geq \int_{0}^{t}\left(\frac{\lambda}{\sigma} \mu_{C}-|a|\right) y^{2}-\beta_{0} \geq \rho \int_{0}^{t} y^{2}-\beta_{0}
$$

which proves the lemma.

Remark 1: The condition (41) exhibits several interesting practical features. It relates the design parameters $(\sigma$, $\lambda)$ as a function of physical drillstring system characteristics such as the nominal $W_{o B}\left(u_{0}\right)$, the rock friction features $\left(\mu_{S}-\mu_{C}\right)$, and the desired rotational velocity $\left(\omega_{d}\right)$. The parameter $\rho$ as shown latter, provides a measure of the convergence rate of the output $y$ to zero.

We are now in a position to establish the main stability result.

Theorem 7.1: Consider the closed-loop system of Figure 13 with $G(s), \Gamma^{*}, \Delta^{*}$ holding the following properties:

(i) $G(s)$ is a PR operator satisfying (31)-(32)

(ii) $\Delta^{*}$ is a passive map satisfying (39)

(iii) $\Gamma^{*}$ is a strictly input passive map satisfying (42), i.e. design parameters are such that the condition (41) holds.

Then, $\left(e^{*}, \tilde{u}^{*}\right)=(0,0)$ is a globally asymptotically stable equilibrium of the considered closed-loop system.

Proof: Let us take the following scalar function:

$$
V(e, \tilde{u})=\frac{1}{2} e^{T} P e+\int_{0}^{t} y\left(\tilde{u}_{2}+\varepsilon y\right)+\left[I(\tilde{u}, y)+\beta_{0}-\rho \int_{0}^{t} y^{2}\right]
$$

From (39) and (42), we have that $V(e, \tilde{u})$ is semi-positive definite.

Computing the time-derivative of $V(e, \tilde{u})$, and using the properties $(i)-(i i i)$ of the theorem, results in:

$$
\dot{V}(e, \tilde{u})=-\frac{1}{2} e^{T} L^{T} L e-\rho y^{2} \leq 0, \forall e, \tilde{u}
$$

Therefore, from last Equation, we have that $y \rightarrow 0$ with a rate depending on the value of $\rho$. The rest of the proof follows from the application of the LaSalle's invariance principle. From Equation (23), we can see that if $y \rightarrow 0$, 

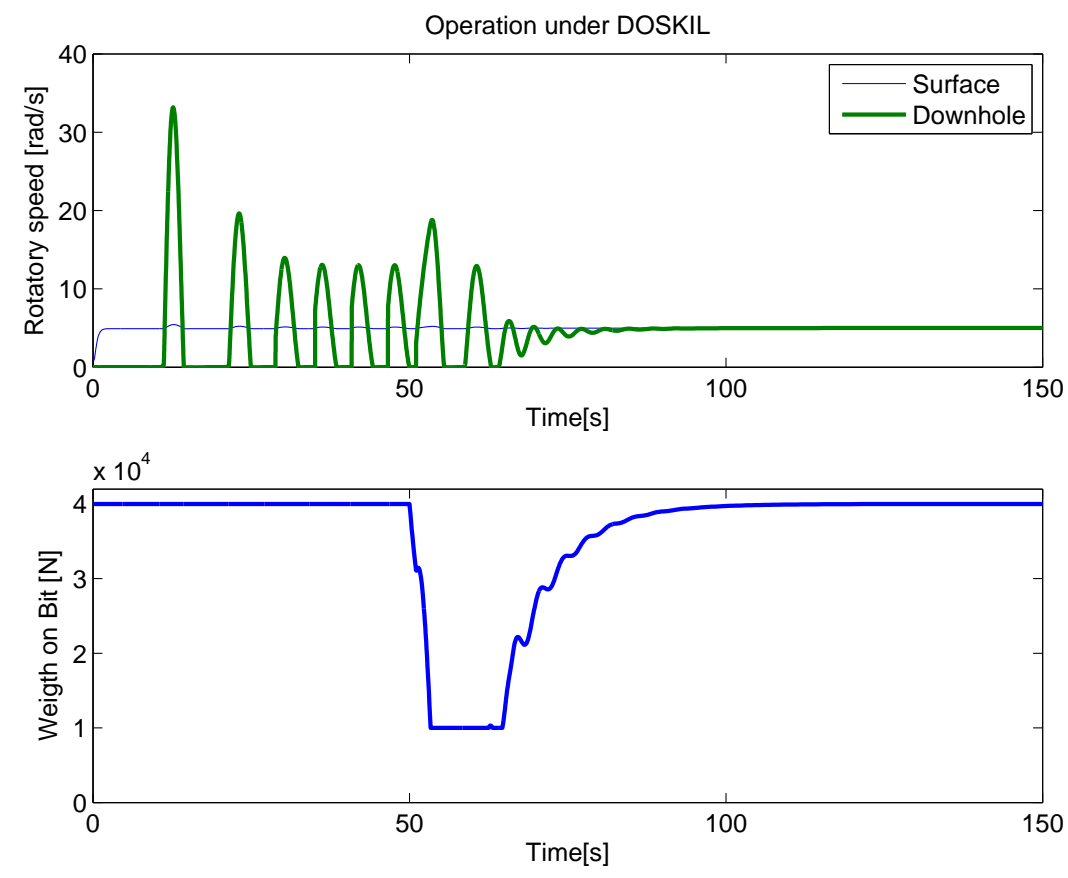

Fig. 15. Simulation of D-OSKIL scheme with $\sigma=0.3$ and $\lambda=2.000$.

together with the fact that $\sigma$ is a positive constant and $\Phi(0)=0$, gives that $\tilde{u} \rightarrow 0$. Finally, this implies that the two last terms in Equation (22) also tends to zero, i.e.

$$
\lim _{t \rightarrow \infty}\left[\mu(y) \tilde{u}+\tilde{\mu}(y) u_{0}\right]=\left[\mu(0) 0+\tilde{\mu}(0) u_{0}\right]=0
$$

since $\mu(0)=\mu^{*}=\mu_{C}$, and $\tilde{\mu}(0)=\mu(0)-\mu^{*}=\mu^{*}-\mu^{*}=0$. Therefore, this results in

$$
\dot{e}=A_{c l} e+H_{c l} \lim _{t \rightarrow \infty}[\cdot]=A_{c l} e
$$

So it can be concluded that $e \rightarrow 0$, and hence that $e^{*}=0$, and $\tilde{u}^{*}=0$ are a globally asymptotically stable equilibria.

\section{Simulation EXAMPLE}

In order to demonstrate the behavior of the proposed adaptive law, simulations of the drillstring system controlled under the D-OSKIL mechanism designed in Section VII-D are shown in Figures 15 and 16. The values ${ }^{3}$ for system model parameters used in the simulations are presented in Table I. In these Figures, the typical profiles in terms on rotatory velocity, both in surface and downhole, and system $W_{o B}$ are shown.

As it can be observed, with the nominal weight $u_{0}=40000 N$, the system is under a sustained oscillation regime. The D-OSKIL mechanism is activated at $t=50 \mathrm{~s}$, and in both cases, the controller is able to extinguish such oscillations, although the $W o B$ profiles obtained are quite different.

\footnotetext{
${ }^{3}$ The numerical values of the drilling system parameters according to a $2000 \mathrm{~m}$ long drillstring have been taken from [29].
} 

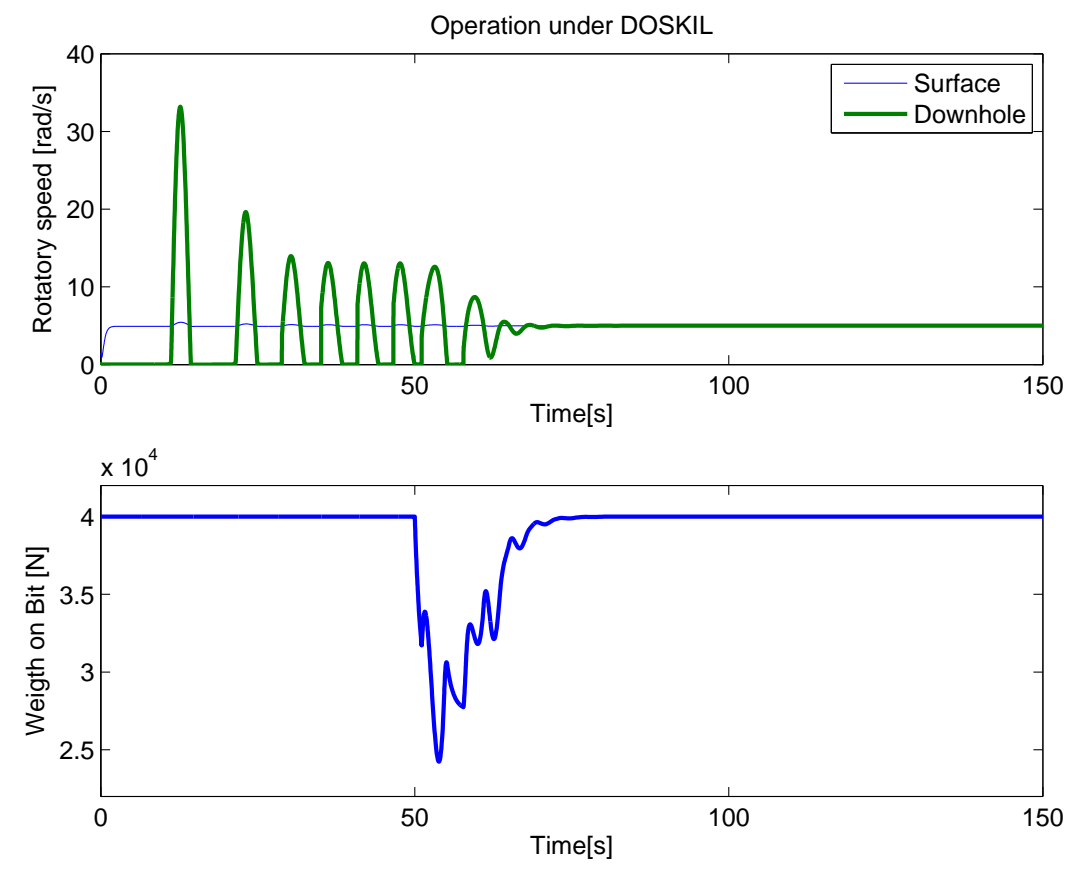

Fig. 16. Simulation of D-OSKIL scheme with $\sigma=1.0$ and $\lambda=2.500$.

In Figure 15 (with control parameters $\lambda=2.000$ and $\sigma=0.3$ ) the D-OSKIl mechanism is able to extinguish the oscillations with a soft evolution in the $W_{o B}$ control signal.

On the other hand, in Figure 16 (with control parameters $\lambda=2.500$ and $\sigma=1.0$ ), the transition from oscillation regime to stabilization period is faster than the one obtained in Figure 15. The stabilization time for the value of $W_{O B}$ is also faster in Figure 16, but in this case some oscillations occur during the transition.

This issue is due to the $\lambda$ value, when large values of the $\lambda$ are chosen a sharp $W_{o B}$ value transition is obtained in the switching instant of time (in our simulations $t=50 \mathrm{~s}$ ).

From a practical point of view, and in order to avoid oscillations in the control signal, the parameter values proposed in Figure 15 seem to be more appropriate.

\section{OBSERVER-BASED DESIGN}

In this section we present some extensions of the previous control which has been studied and designed under the hypothesis of the measure of the bit rotational velocity $\dot{\varphi}_{b}$. In this section we first provide an alternative way to get this measure trough a state observer.

\section{A. Observer Design}

The observer is designed on the basis of open-loop equation (8). That is on

$$
\begin{aligned}
\dot{x} & =A x+B v+(H u) \cdot \mu \\
y_{o} & =C_{o} x=\dot{\varphi}_{r}
\end{aligned}
$$


Following [35] we propose to use the following observer based on the observation of the rotary angular velocity $y_{o}=\dot{\varphi}_{r}:$

$$
\begin{aligned}
\dot{\hat{x}} & =A \hat{x}+B v+(H u) \cdot \hat{\mu}+\left[K_{o}+\beta \Gamma \Gamma^{T} C_{o}^{T}\right]\left(y_{o}-C_{o} \hat{x}\right) \\
\dot{\hat{\mu}} & =\beta \Gamma^{T} C_{o}^{T}\left(y_{o}-C_{o} \hat{x}\right) \\
\dot{\Gamma} & =\left[A-K_{o} C_{o}\right] \Gamma+(H u)
\end{aligned}
$$

where: $\Gamma$ is a $(3 \times 1)$ time-varying vector, $\beta>0$ is a positive scalar, $\hat{x}$, and $\hat{\mu}$ are the state and friction coefficient estimates respectively, $y_{o}$ is the measured output, $K_{o}$ is a $(3 \times 1)$ observer vector gain, and $C_{o}=[0,1,0]$. Note that $C_{o} \neq C$.

The observer not only provides an estimate for the bit rotational velocity $\hat{x}_{3}$, but also provide an estimate of the friction coefficient $\hat{\mu}$ which can be useful for other monitoring purposes. The observer, as indicated in [35], results in a globally exponentially stable observer providing the following hypothesis hold:

- $\mu$ is constant

- Exists a matrix $K_{o}$ such that $\left(A-K_{o} C_{o}\right)$ is strictly stable matrix, i.e. The constant pair $\left(A, C_{o}\right)$ is detectable.

- $u(t)$ is persistently exciting, i.e. $\exists \delta, T>0$ such that the following inequality is satisfied:

$$
\int_{t}^{t+T} \Gamma(\tau)^{T} C_{o}^{T} C_{o} \Gamma(\tau) d \tau>\delta>0
$$

Let comment the practical implication of the previous hypothesis.

The first hypothesis assumes that the friction coefficient is constant, or eventually slow-time variant ${ }^{4} \dot{\mu} \approx 0$. Note that this approximation is often assumed in the context of observer design with unknown inputs, but also in the context of adaptive control. Here this hypothesis means that the rate of variation of the rock friction coefficient does not exhibit substantial changes during the drill-operation. Even if the drilled surfaces may have different friction characteristics, the rate of penetration (drilling-speed) remains small.

The second hypothesis correspond to the necessity observation property need to build the observed. By inspecting this condition, we can see that the system observability is invariant with respect the matric $A$ and $C_{o}$.

Finally, the last property is necessary to the observer to converge. Note that as the "adaptation" is done under a single parameter $\mu$, the required condition is weak and will be simple to fulfill. To see this note that the pair $\left(A-K_{o} C_{o}, H\right)$ is controllable and the pair $\left(A-K_{o} C_{o}, C_{o}\right)$ is observable, then the persistently exciting condition is easily verified if the $\mathrm{WoB}$ force is not equal to zero, i.e. $u(t)>0$. A detailed justification of this can be found in [11].

\section{B. Simulation with the observed-based controller}

The original controller has the form

$$
\dot{\tilde{u}}=-\sigma \tilde{u}+\lambda\left(x_{3}-\omega_{d}\right) \cdot \operatorname{sgn}\left(\mu\left(x_{3}-\omega_{d}\right)\right)
$$

\footnotetext{
${ }^{4}$ In this case, it can also be shown that stability (not asymptotic) follows is preserved
} 

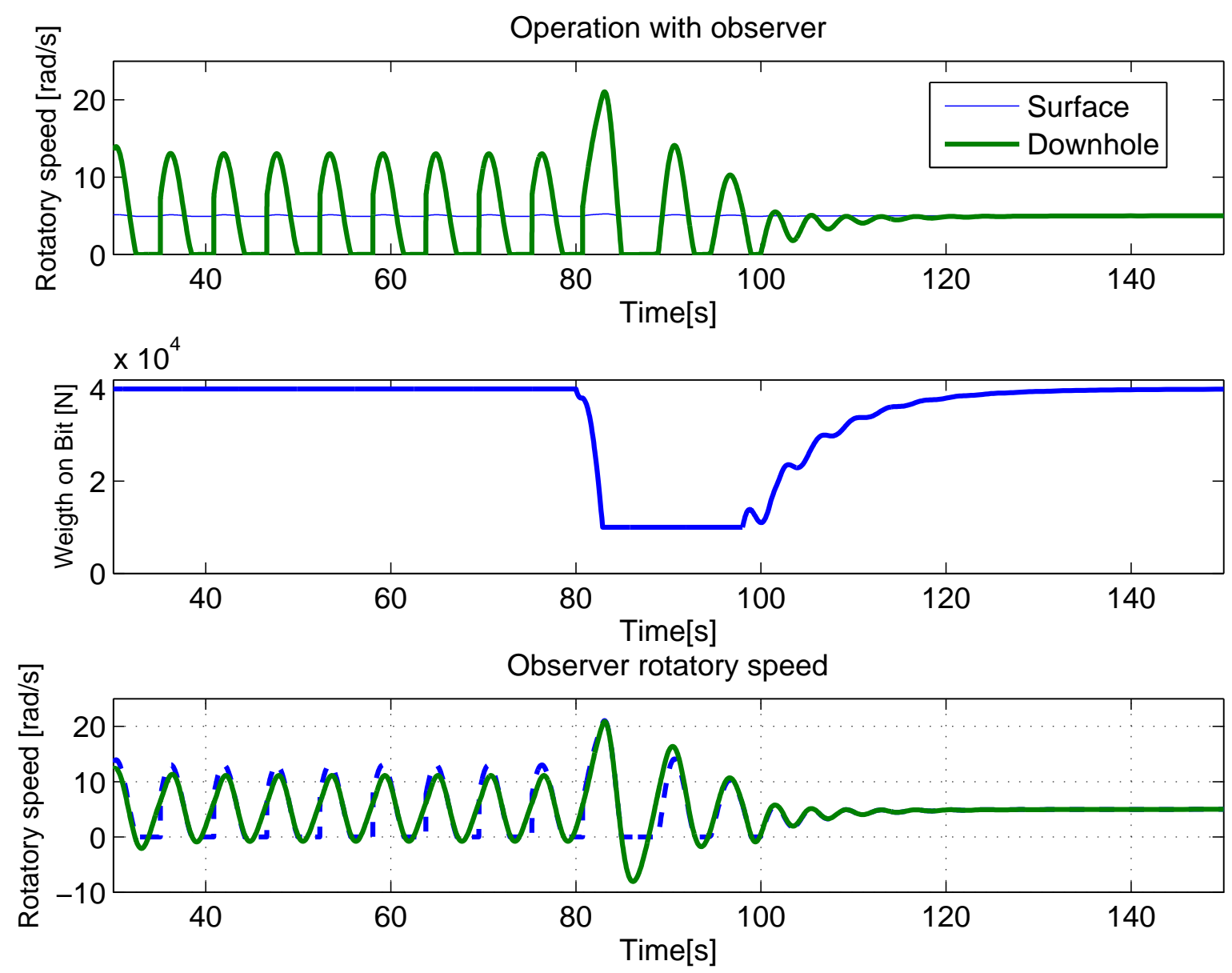

Fig. 17. Simulation of D-OSKIL scheme with observer. The D-OKILL mechanisms is activated at after 80 sec. The upper plots show the Surface and the downhole rotational speed. The middle plots show the time-evolution of the weight on bit. It has been saturated for efficiency reasons at $1000 N$. The lower plots shows the downhole velocity and its estimated.

replacing $x_{3}$ by its estimate $\hat{x}_{3}$, and noting that $\operatorname{sgn}\left(\mu\left(x_{3}-\omega_{d}\right)\right)=\operatorname{sgn}\left(x_{3}-\omega_{d}\right)$, we get the final, quite simple, control law structure to be implemented together with the observer,

$$
\left.\dot{\tilde{u}}=-\sigma \tilde{u}+\lambda \mid \hat{x}_{3}-\omega_{d}\right) \mid
$$

In Figure 17, we repeat the experiment shown previously in Figure 15 (with the parameters given in Table I) with the observed-based control law instead. The additional parameter for the observer used in this simulation where: $\beta=0.05$ and $K_{o}=[-26.8125 ; 4.5928 ;-10.4562]$. As the figure shows, the D-OSKIL mechanism works well in this case. The behavior obtained in this figure and in Figure 15 are quite similar. Note that the observer can tolerate a certain amount of uncertainty on the knowledge of the physical parameters as demonstrated by the authors in [35]. The figure at the bottom shows the comparison between the downhole rotatory speed and the signal provided by the observer. 
TABLE I

PARAMETER VALUES USED IN SIMULATION OF FiguRE 15.

\begin{tabular}{|c|r|c||}
\hline Parameter & Value & Unit \\
\hline$J_{r}$ & 2122 & {$\left[\mathrm{Kg} \cdot \mathrm{m}^{2}\right]$} \\
$J_{b}$ & 374 & {$\left[\mathrm{Kg} \cdot \mathrm{m}^{2}\right]$} \\
$k$ & 473 & {$[\mathrm{Nm} / \mathrm{rad}]$} \\
$c$ & 23.2 & {$\left[1 / \mathrm{s}^{2}\right]$} \\
$d_{r}$ & 425 & {$[1 / \mathrm{s}]$} \\
$d_{b}$ & 50 & {$[1 / \mathrm{s}]$} \\
$\mu_{C}$ & 0.3 & {$[-]$} \\
$\mu_{S}$ & 0.35 & {$[-]$} \\
$v_{s}$ & 0.01 & {$[\mathrm{rad} / \mathrm{s}]$} \\
$u_{0}$ & 40000 & {$\left[\mathrm{Kg} \cdot \mathrm{m} / \mathrm{s}^{2}\right]$} \\
$\omega_{d}$ & 5 & {$[\mathrm{rad} / \mathrm{s}]$} \\
$k_{1}$ & 15725 & {$[\mathrm{Nm} / \mathrm{rad} / \mathrm{s}]$} \\
$k_{2}$ & 30576 & {$[\mathrm{Nm} / \mathrm{rad}]$} \\
$k_{3}$ & 194 & {$[\mathrm{Nm} / \mathrm{rad} / \mathrm{s}]$} \\
\hline
\end{tabular}

\section{CONCLusions}

We have proposed to use the weight on the bit (WoB) force as an additional control variable to extinguish limit cycles when they occur. An adaptation law, named D-OSKIL, of the oscillation killer (OSKIL) mechanism, to oil well drillstring systems has been proposed. An approximate analysis based on the bias describing function did provide a good insight of the slip-stick behavior and in the control design.

We have then presented a stability analysis of a variant of the D-OSKIL control mechanism, introduced in [7], to remove stick-slip oscillations in drillstring systems. The presented stability analysis based on passivity has shown that this algorithm is globally asymptotically stable. Simulations applying such an algorithm showed that stick-slip oscillations can be effectively eliminated.

The implementation of the proposed control algorithm requires the sign of a friction torque on the bit, $\mu(y)$, which can be computed if the sign of rotational velocity measured at the bit is known. It has also been shown in the paper, that the measurement of this velocity can be undertaken by the proper design of an nonlinear observer, without jeopardizing the performance of the closed-loop system.

\section{ACKNOWLEDGMENT}

The authors would like to acknowledge MCYT-FEDER for funding this work under grants DPI2004-06419, HF2003-0237 and SAB2003-0085. Thanks for funding are also due to the program PICASSO No. 07261YJ(EGIDE), of the French Ministry of Foreign Affaires. 


\section{APPENDIX}

\section{A. TUning METhOD FOR CONTROLler GAins}

From the system model Equations (1) and (2), and defining a new variable $z$ and constant $\epsilon$ in the way:

$$
z=k\left(\varphi_{r}-\varphi_{b}\right), \quad \epsilon^{2}=\frac{1}{k}
$$

the $\varphi_{r}$ and $z$ dynamics are:

$$
\begin{aligned}
J_{r} \ddot{\varphi}_{r} & +c \epsilon^{2} \dot{z}+z+d_{r} \dot{\varphi}_{r}=v \\
\epsilon^{2} \ddot{z} & =-\frac{z}{J_{e q}}-\epsilon^{2}\left(\frac{c}{J_{e q}}+\frac{d_{b}}{J_{b}}\right) \dot{z}+ \\
& +\left(\frac{d_{b}}{J_{b}}-\frac{d_{r}}{J_{r}}\right) \dot{\varphi}_{r}+\frac{v}{J_{r}}+\frac{T o B}{J_{b}}
\end{aligned}
$$

Since the torsional stiffness $k$ is usually very large, the assumption $\epsilon=0$ can be made, and so:

$$
\begin{aligned}
& J_{r} \ddot{\varphi}_{r}+z+d_{r} \dot{\varphi}_{r}=v_{s} \\
& 0=-\frac{z}{J_{e q}}+\left(\frac{d_{b}}{J_{b}}-\frac{d_{r}}{J_{r}}\right) \dot{\varphi}_{r}+\frac{v_{s}}{J_{r}}+\frac{T o B}{J_{b}}
\end{aligned}
$$

where the control signal is supposed to be split in slow $\left(v_{s}\right)$ and fast $\left(v_{f}\right)$ modes:

$$
v=v_{s}+\epsilon v_{f}
$$

Therefore, solving Equation (55):

$$
z=J_{e q}\left[\left(\frac{d_{b}}{J_{b}}-\frac{d_{r}}{J_{r}}\right) \dot{\varphi}_{r}+\frac{v_{s}}{J_{r}}+\frac{T o B}{J_{b}}\right]
$$

And so, Equation (54) results in:

$$
\ddot{\varphi}_{r}+\left(\frac{d_{b}+d_{r}}{J_{r}+J_{b}}\right) \dot{\varphi}_{r}=\frac{1}{J_{r}+J_{b}} v_{s}-\frac{1}{J_{r}+J_{b}} T o B
$$

By supposing $v_{s}=\left(k_{1}+\frac{k_{2}}{s}\right)\left(\omega_{d}-\dot{\varphi}_{r}\right)$, and defining a new variable:

$$
\dot{\tilde{\varphi}}_{r}=\omega_{d}-\dot{\varphi}_{r} \Rightarrow \tilde{\varphi}_{r}=\frac{\omega_{d}-\dot{\varphi}_{r}}{s}, \quad \ddot{\tilde{\varphi}}_{r}=-\ddot{\varphi}_{r}
$$

We have (supposing $\left.\left(d_{b}+d_{r}\right) \ll\left(J_{r}+J_{b}\right)\right)$ :

$$
\ddot{\tilde{\varphi}}_{r}+\left(\frac{k_{1}}{J_{r}+J_{b}}\right) \dot{\tilde{\varphi}}_{r}+\left(\frac{k_{2}}{J_{r}+J_{b}}\right) \tilde{\varphi}_{r}=\frac{T o B}{J_{r}+J_{b}}
$$

And so, by imposing some damping $\left(\delta_{r}\right)$ and natural frequency $\left(\omega_{n}\right)$ in the closed loop dynamics $T o B \mapsto \tilde{\varphi}_{r}$, the values of $k_{1}$ and $k_{2}$ can be computed from:

$$
\begin{gathered}
k_{1}=\left(J_{b}+J_{r}\right) \cdot\left[2 \delta_{r} \omega_{n}-\frac{d_{b}+d_{r}}{J_{b}+J_{r}}\right] \\
k_{2}=\left(J_{b}+J_{r}\right) \omega_{r}^{2}
\end{gathered}
$$


On the other hand, by substituting the controller structure in Equation (53), the following expression is obtained:

$$
\begin{aligned}
\epsilon^{2} \ddot{z}= & -\frac{z}{J_{e q}}-\epsilon^{2}\left(\frac{c}{J_{e q}}+\frac{d_{b}}{J_{b}}\right) \dot{z}+\left(\frac{d_{b}}{J_{b}}-\frac{d_{r}}{J_{r}}\right) \dot{\varphi}_{r}+ \\
& +\frac{v_{s}}{J_{r}}+\frac{\epsilon}{J_{r}} v_{f}+\frac{T o B}{J_{b}}
\end{aligned}
$$

Due to two-time scales separation, it can be assumed that the slow variable has reached its steady state value respecting to the variations of the fast variable $z$, and so, rewriting Equation (61) as:

$$
\epsilon^{2} \ddot{z}=-\frac{z}{J_{e q}}-\epsilon^{2}\left(\frac{c}{J_{e q}}+\frac{d_{b}}{J_{b}}\right) \dot{z}+\frac{\epsilon}{J_{r}} v_{f}+\frac{T o B}{J_{b}}+\rho\left(\dot{\varphi}_{r}^{*}, \varphi_{r}^{*}\right)
$$

where the superscript ${ }^{*}$ means steady-state value, and:

$$
\rho\left(\dot{\varphi}_{r}^{*}, \varphi_{r}^{*}\right)=\left(\frac{d_{b}}{J_{b}}-\frac{d_{r}}{J_{r}}\right) \dot{\varphi}_{r}^{*}+\frac{v_{s}\left(\dot{\varphi}_{r}^{*}, \varphi_{r}^{*}\right)}{J_{r}}=\frac{z^{*}}{J_{e q}}-\frac{T o B}{J_{b}}
$$

By rewriting Equation (62) in fast error coordinates $\zeta=z-z^{*}$, we have:

$$
\ddot{\zeta}+\left(\frac{c}{J_{e q}}+\frac{d_{b}}{J_{b}}\right) \dot{\zeta}+\frac{1}{\epsilon^{2} J_{e q}} \zeta=\frac{1}{J_{r} \epsilon} v_{f}
$$

Taking $v_{f}=-k_{3} \dot{\zeta}$ :

$$
\ddot{\zeta}+\left(\frac{c}{J_{e q}}+\frac{d_{b}}{J_{b}}+\frac{k_{3}}{J_{r} \epsilon}\right) \dot{\zeta}+\frac{1}{\epsilon^{2} J_{e q}} \zeta=0
$$

and so, imposing certain damping value for torsion dynamics $\left(\delta_{t o r}\right)$, the value of $k_{3}$ can be computed:

$$
k_{3}=\frac{J_{r}}{\sqrt{k}} \cdot\left[2 \delta_{t o r} \sqrt{k} J_{e q}-\frac{c}{J_{e q}}-\frac{d_{b}}{J_{b}}\right]
$$

B. Computation of the Functions $N_{0}$, And $N_{1}$

$$
\begin{gathered}
N_{0}\left(A_{0}, y_{0}\right)=\frac{1}{2 \pi y_{0}} \int x(t) d(\theta) \\
N_{1}\left(A_{0}, y_{0}\right)=\frac{1}{\pi A_{0}} \int x(t) \cos (\theta) d(\theta) \\
N_{0}\left(A_{0}, y_{0}\right)=\frac{2 F_{s}}{y_{0}} g\left(\frac{y_{0}}{A_{0}}\right)-\frac{A_{0}\left(F_{s}-F_{c}\right)}{2 y_{0} v_{s}} . \\
\cdot\left[f_{0}\left(\frac{v_{s}+y_{0}}{A_{0}}\right)-f_{0}\left(\frac{v_{s}-y_{0}}{A_{0}}\right)\right] \\
N_{1}\left(A_{0}, y_{0}\right)=\frac{4 F_{s}}{\pi A_{0}} D_{1}\left(\frac{y_{0}}{A_{0}}\right)-\frac{\left(F_{s}-F_{c}\right)}{2 v_{s}} . \\
\cdot\left[f_{1}\left(\frac{v_{s}+y_{0}}{A_{0}}\right)+f_{1}\left(\frac{v_{s}-y_{0}}{A-0}\right)\right]
\end{gathered}
$$


with:

$$
\begin{gathered}
g(x)=\left\{\begin{array}{cc}
\frac{\arcsin (x)}{\pi} & |x| \leq 1 \\
\frac{-1}{2} & x<-1 \\
\frac{1}{2} & x>1
\end{array}\right\} \\
f_{0}(x)=\left\{\begin{array}{cc}
\frac{2}{\pi}\left(x \arcsin (x)+\sqrt{1-x^{2}}\right) & |x| \leq 1 \\
|x| & |x|>1
\end{array}\right\} \\
f_{1}(x)=\left\{\begin{array}{cc}
\frac{2}{\pi}\left(\arcsin (x)+x \sqrt{1-x^{2}}\right) & |x| \leq 1 \\
-1 & x<-1 \\
1 & |x| \leq 1 \\
\sqrt{1-x^{2}} & |x|>1
\end{array}\right\} \\
D_{1}(x)=\left\{\begin{array}{cc}
0 & \mid x>1 \\
0
\end{array}\right.
\end{gathered}
$$
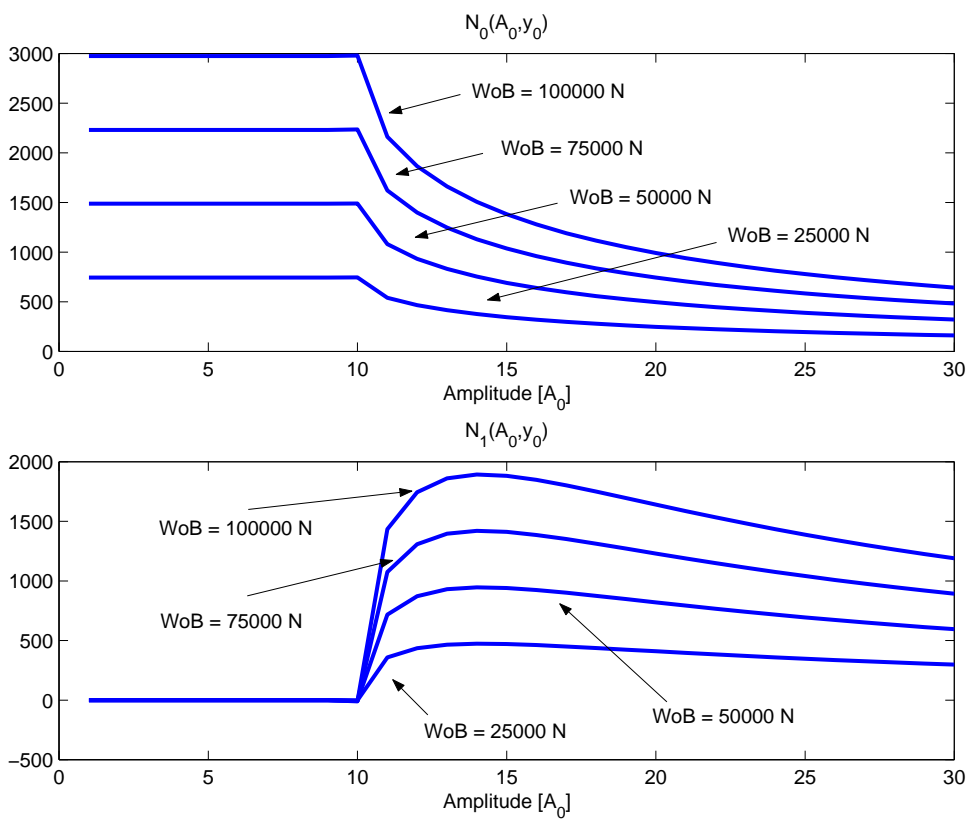

Fig. 18. $N_{0}\left(A_{0}, y_{0}\right)$ and $N_{1}\left(A_{0}, y_{0}\right)$ functions in the case that $y_{0}=5 \mathrm{rad} / \mathrm{s}$.

\section{REFERENCES}

[1] Abbasssian, F., Dunayevsky, V.A. Application of Stability Approach to Torsional and Lateral Bit Dynamics. SPE Drilling and Completion, 13(2):99-107, 1998.

[2] Abdulgalil, F., Siguerdidjane, H. Nonlinear Control Design for Suppressing Stick-Slip in Oil Well Drillstrings. 5th Asian Control Conference, pages 1276-1281, 2004.

[3] Armstrong-Hélouvry, B. Stick-Slip Arising from Stribeck Friction. IEEE International Conference on Robotics and Automation, 2:13771382, 1990. 
[4] Atherton D.P. Nonlinear Control Engineering. Describing Function: Analysis and Design. Van Nostrand Reinhold, first edition, 1982.

[5] Brett, J. F. The Genesis of Torsional Drillstring Vibrations. SPE Drilling Engineering, September:168-174, 1992.

[6] Canudas-de-Wit, C., Aracil, J., Gordillo, F., Salas, F. The oscillations killer: a mechanism to eliminate undesired limit cycles in nonlinear systems. 44th IEEE Conference on Decision and Control, and the European Control Conference, CDC-ECC'05, Seville (Spain), 2005.

[7] Canudas-de-Wit, C., Corchero, M.A., Rubio, F.R., Navarro-Lopez, E.M. D-OSKIL: A new mechanism for suppressing stick-slip in oil well drillstrings. 44th IEEE Conference on Decision and Control, and the European Control Conference, CDC-ECC'05, Seville (Spain), 2005.

[8] Canudas-de-Wit C., Olsson H., Astrom K.J. and Lischinsky P. A new model for control of systems with friction. IEEE Transactions on Automatic Control, 40(3), 1995.

[9] Canudas-de-Wit, C., Siciliano, B., Bastin, G. Theory of Robot Control. Springer, first edition, 1996.

[10] Christoforou, A. P., Yigit, A. S. Fully coupled vibrations of actively controlled drilstrings. Journal of Sound and Vibration, 267:1029-1045, 2003.

[11] Dolcini, P., Bechart, H., Canudas-de-Wit, C. Observer-based Optimal Control of Dry Clutch Engagement. 44th IEEE Conference on Decision and Control, and the European Control Conference, CDC-ECC'05, Seville (Spain), pages 440-445, 2005.

[12] Dupont P., Hayward V., Armstrong B. and Altpeter F. Single state elastoplastic friction models. IEEE Transactions on Automatic Control, 47(5), 2002.

[13] Halsey, G. W., Kyllingstad, A., Kylling, A. Torque Feedback Used to Cure Slip-stick Motion. in the 63rd SPE Annual Technical Conference and Exhibition, pages 277-282, 1988.

[14] Harris, C.M., Piersol, A.G. Harris's Shock and Vibration Handbook. McGraw-Hill, 5th edition, 2002.

[15] Jansen, J. D., van den Steen, L. Active Damping of Self-excited Torsional Vibrations in Oil Well Drillstrings. Journal of Sound and Vibration, 179(4):647-668, 1995.

[16] Khalil, H.K. Nonlinear Systems. Prentice-Hall, 2nd edition, 1996.

[17] Kyllingstad, A., Halsey, G. W. A Study of Slip/stick Motion of the Bit. SPE Drilling Engineering, pages 369-373, 1988.

[18] Lampaert V., Swevers J. and Al-Bender F. Modification of the Leuven integrated friction model structure. IEEE Transactions on Automatic Control, 47(4), 2002.

[19] Leine R.I., van Campen D.H., Keultjes W.J.G. Stick-slip whirl interaction in drillstring dynamics. Journal of Vibration and Acoustics, 124:209-220, 2002.

[20] Lin, Y.Q., Wang, Y.H. Stick-slip Vibration of Drill Strings. Journal of Engineering for Industry, 113:38-43, 1991.

[21] Navarro-Lopez, E.M. Notas acerca del modelado, analisis y control de las vibraciones mecanicas en una sarta de perforacion. Instituto Mexicano de Petroleo, programa de investigación en matematicas aplicadas y computacion edition, 2003.

[22] Navarro-Lopez, E.M. and Suarez-Cortez, R. Modelling and analysis of stick-slip behaviour in a drillstring under dry friction. Congress of the Mexican Association of Automatic Control, pages 330-335, 2004.

[23] Navarro-Lopez, E.M. and Suarez-Cortez, R. Practical approach to modelling and controlling stick-slip oscillations in oilwell drillstrings. IEEE International Conference on Control Applications, pages 1454-1460, 2004.

[24] Navarro-Lopez, E.M. and Suarez-Cortez, R. Vibraciones mecánicas en una sarta de perforación: problemas de control. Revista Iberoamerica de Automática e Informática Industrial, 2(1), 2005.

[25] Pavone D. R., Desplans J.P. Application of High Sampling Rate Downhole Measurements for Analysis and Cure of Stick-slip in Drilling. in SPE Annual Technical Conference and Exhibition, SPE 28324:335-345, 1994.

[26] Rabia, H. Oil Well Drilling Engineering: Principle and Practice. Graham \& Trotman, 1985.

[27] Richard T. Self-excited Stick-slip Oscillations of Drag Bits. Ph.D. thesis, 2001.

[28] Sananikone, P., Kamoshima, O., White, D.B. A Field Method for Controlling Drillstring Torsional Vibrations. in IADC/SPE Drilling Conference, IADC/SPE 23891:443-452, 1992.

[29] Serrarens A.F.A, van de Molengraft M.J.G., Kok J.J. and van den Steen L. $H_{\infty}$ control for suppressing stick-slip in oil well drillstrings. IEEE Control Systems Magazine, 18(2), 1998.

[30] Shorten, R., C. King. Spectral conditions for positive realness of single-input-single-output systems. IEEE Transactions on Automatic Control, 49(10), 2004. 
[31] Spanos, P.D., Chevallier, A.M., Politis N.P. and Payne M.L. Oil well drilling: A vibrations perspective. The Shock and Vibration Digest, 35(2):81-99, 2003.

[32] van de Vrande, B.L., van Campen, D.H., de Kraker, A. An Approximate Analysis of Dry-friction-induced Stick-slip Vibrations by a Smoothing Procedure. Nonlinear Dynamics, 19:157-169, 1999.

[33] Vidyasagar, M. Nonlinear Systems Analysis. Prentice-Hall, second edition, 1993.

[34] William Ranney, M. Offshore Oil Technology Recent Development. Noyes Data Corporation, 1979.

[35] Zhang, Q. Adaptive observer for MIMO linear time varying systems. INRIA report, No.4111, 2001. 